UNIVERSIDADE DE SÃO PAULO

INSTITUTO DE FISICA E QUIMICA DE SÃO CARLOS

EFEITOS DE CONDIÇÃO DE CONTORNO SOBRE O ESPECTRO DA CADEIA QUAN TICA DE BLUME-CAPEL

Deborah Brasil Balbão

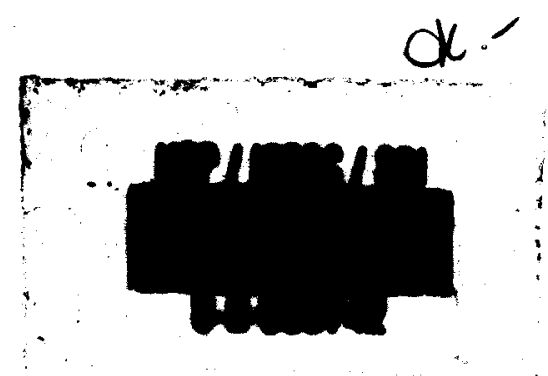

Dissertação apresentada ao Instituto de Física e Química de sãc Carlos, da Universidade de São Paulo, para obtenção do título de Mestre em Física Básica

Orientador: Prof.Dr.Josē Roberto Drugowich de Felício

São Carlos

$\begin{array}{llll}1 & 9 & 8 & 7\end{array}$ 
MEMBROS DA COMISSÃO JULGADORA DA DISSERTAÇAO DE MESTRADO DE Deborah Brasil Balbão APRESENTADA AO INSTITUTO DE FISICA E QUIMICA DE SAO CARLOS, DA UNIVERSI DADE DE SAO PAULO, EM $20 \quad D E$ E março DE $198 \underline{7}$.

COMISSAO JULGADORA:

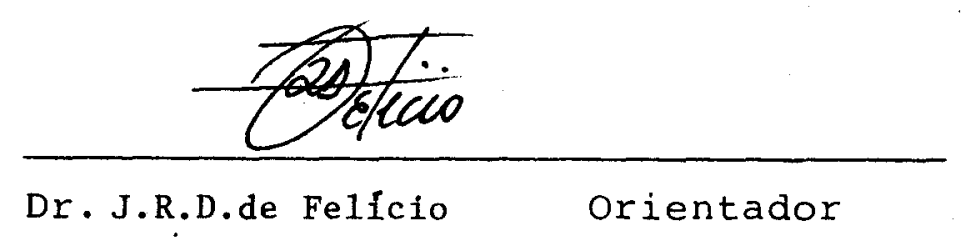

Robento wer ce iz Dr. Roberto Nicolau Onody




Agradeço

a ti, Zezão, pelo amor, pela força, pela paciēncia e por ser parte de mim agora e sempre;

ao Chico, ao Lula, ao Jaime, ao Val ter, ao Almir, ao Gavião, ao Giomal e à Dayse pela amizade e pela luz que me fizerem ver e viver;

à música, à chuva, ao sol, à vida e a vocês: Dani, Kica, Clarissa, Alexandre, Rafael, Tia Dulce, Tia Célia e Vó sōnia;

Finalmente, agradeço ao Drugo pelo incentivo, pelo apoio e pela amizade, sem os quais este trabalho não teria chegado até aqui. 
$\AA$

minha família

Ao Josē Fernando e

ao meu amigo

Drugowich 
INDICE

RESUMO. ........................... I



CAPITULO I - INTRODUÇÃO...................... 1

CAPITULO II- INVARIÂNCIA CONFORME EM 2 DIMENSÕES....... 5

1. Invariância Conforme............ 5

2. Espectro................... 8

3. Operadores primārios............... 11

4. Invariäncia Conforme e a Algebra de Vira soro......................... 14

5. Aplicação ao Modelo de Ising em Campo Transverso................... 22

CAPITULO III-O MODELO DE BLUME-CAPEL............ 33

1. o Modelo.................... 33

2. o Diagrama de Fases............ 40

3. Cálculo do Número de Anomalia Confor me $c . \ldots \ldots \ldots \ldots \ldots \ldots \ldots \ldots \ldots, 41$

CAPITULO IV -EFEITO DAS CONDIÇÕES DE CONTORNO SOBRE O ESPECTRO DO MODELO DE BLUME-CAPEL........ 45

1. Condições Periódicas de Contorno....... 46

2. Condições Antiperiōdicas de Contorno.... 49

3. Condição Livre de Contorno.......... 54

4. Condição Fixa de Contorno.......... 55

5. Comentärios Finais.............. 58

APENDICE A - Regras de Soma de Inversão............. 59

APENDICE B - o Método de Lanczos.................. 63

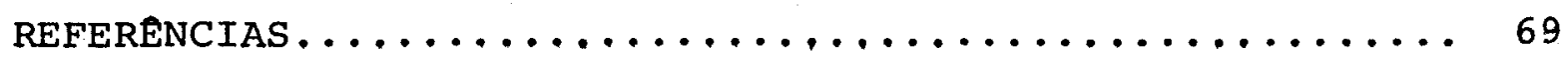


RESUMO

Nesse trabalho nós apresentamos o estudo numérico do espectro da cadeia quântica de Blume-Capel no ponto tricrítico (aquele que separa as linhas de transição de la. e 2a.ordem). Os resultados obtidos foram comparados com as previsões da invariância conforme que permitiram construir o espectro a partir da combinação das dimensōes de escala associadas aos operadores da teoria. O parâmetro que caracteriza as classes de universalidade, chamado nümero de anomalia conforme, foi calculado através das correções à energia do estado fundamental de cadeias finitas. Finalmente várias condições de contorno foram utilizadas para investigar a associaçäo entre os $\underline{0}$ peradores e suas dimensões, bem como para calcular os expoen tes críticos das funções de correlação na superfície. 
ABSTRACT

We calculated the conformal anomaly number of the Blume-Capel quantum chain via finite-size corrections to the ground-state energy. In addition we compute, at the tricritical point, the lowest excitations of the spectrum to be compared with the theoretical description in terms of the irreducible representations of the corresponding Virasoro Algebra. Several boundary conditions are analyzed. In particular, surface exponents are obtained by using free boundary conditions. 
Este trabalho teve o apoio financelro da FAPESP do CNPq. 
CAP ITULO I

INTRODUÇÃO

A utilidade da invariância conforme em câlculos de Mecânica Estatistica, apesar de sugerida hã muitos anos por Polyakov ${ }^{1}$, só muito recentemente foi reconhecida de forma definitiva. Curiosa mente essá redescoberta aconteceu por acaso, quando em seus cālculos numëricos Luck ${ }^{2}$, Derrida e de sèze ${ }^{3}$ encontraram uma relação dí reta entre as amplitudes do comprimento de correlação e a dimensão anômala do operador cuja correlação estava sendo considerada. As coincidências numēricas foram estendidas por Nigthingale e Blyte 4 alēm de Privman e Fisher ${ }^{5}$, mas foi John Cardy $^{6,7}$ quem conseguiu ex plicar o fato (usando o princípio da invariância conforme) e inaugurar uma nova etapa para o "Finite-Size Scaling" (F.S.S.).

Esse breve histórico da união F.S.S. + invariāncia con forme omite, no entanto, um avanço muito importante conseguido por Belavin, Polyakov e Zamolodchikov ${ }^{8}$ (BPZ) no inicio de 1984. Nesse trabalho eles mostraram que os campos locais, de uma teoria confor malmente invariante em duas dimensões ${ }^{*}$, podem ser classificados de acordo com as representações irredutiveis da ālgebra de virasoro e que todas as funções de correlação podem ser construídas a partir dos "blocos conforme", que são por sua vez completamente determina dos pela invariância conforme. Em particular são obtidas todas as dimensões anōmalas (expoentes cr1ticos) dos operadores presentes na teoria. Vale a pena mencionar, no entanto, que a completa deter

* Para dimensão $d>2$ o grupo conforme tem dimensão finita e consiste de translações, rotações, dilatações e transformações conforme especiais. 
minação das funções de muitos pontos só pode ser feita se as repre sentações mencionadas forem tais que conduzam ao desaparecimento de uma certa quantidade chamada determinante de $\mathrm{Kac}^{9}$. Para fechar - trabalho de BPZ faltava apenas explicar, então, porque se deve ria escolher apenas as particulares representações que anulam o de terminante de Kac. Foi exatamente isso que Friedan, Qiu e Shenker ${ }^{10}$ conseguiram fazer, invocando o argumento da unitariedade (a condição que nenhum dos estados gerados tenha norma negativa). Dessa forma, ficou provado que se a carga central $\underline{c}$, que é o número que parametriza a álgebra de Virasoro, for menor que 1, as únicas re presentações possiveis são dadas pela fórmula de Kac.

Fica claro, do exposto acima, que o número adimensional $\underline{c}$, também chamado número de anomalia conforme, praticamente nos conduz aos expoentes críticos de uma certa classe de universalidade. Ressalve-se, entretanto, que se esse nūmero for determinado por argumentos muito gerais (por exemplo: à Lagrangeana $\mathcal{L}=$ $=\frac{1}{2} \psi \frac{\partial \psi}{\partial \bar{z}}+\frac{1}{2} \bar{\psi} \frac{\partial \psi}{\partial z}$ está associado o tensor momento-energia $\Gamma(z)=$ $=-\frac{1}{2}: \psi(z) \frac{\partial}{\partial z} \psi(z)$ : que satisfaz a álgebra de virasoro com $c=$ $=1 / 2$ ) corre-se o risco de não se saber a que modelo de mecânica es tatística os índices críticos obtidos corresponderão. A situação , nesse caso, conforme palavras de Cardy en seu artigo de revisãol1, "é análoga àquela de um fisico atômico que classifica todos os pos síveis elementos pelos seus apropriados números quânticos, mas pre cisa apelar a outras informações para saber qual deles é incolor, inodoro, adequado à respiração dos vertebrados". Em virtude dessa dificuldade foi muito importante a conexão, recentemente descoberta por Blotte et al ${ }^{12}$ além de I.Affleck, entre a carga central $c$ e - maior autovalor da matriz de transferência de um sistema, finito em uma direção e infinito na outra (simetria de cinta). De acordo com esses autores , a correção de "scaling" à energia livre por 
sítio, de um sistema $L \times \infty$, na temperatura crítica do análogo infinito é universal e vale (no caso de condições periódicas de contor no) $\frac{\pi c}{6}$, sendo $\subseteq$ a carga central. No caso de se encontrar um valor menor do que 1 para essa quantidade todas as dimensões anômalas po dem ser obtidas através da fórmula de $\mathrm{Kac}^{9}$

$$
h_{p, q}=\frac{[p(m+1)-q m]^{2}-1}{4 m(m+1)} \quad 1 \leqslant q \leqslant p \leqslant m-1
$$

onde m satisfaz a equação

$$
c=1-\frac{6}{m(m+1)} .
$$

Os comentārios anteriores podem dar a impressão de que o estudo do autovalor dominante da matriz de transferência lou do es tado fundamental do hamiltoniano associado $\hat{\mathrm{H}}=-\frac{1}{\tau} \ln \hat{T}$, seria suficiente para o completo entendimento de um modelo, uma vez que is so permitiria calcular o número $\underline{c}$, e consequentemente as dimensöes dos operadores. Entretanto, nesse esquema fica faltando identifi car as dimensões, com os seus respectivos operadores, tarefa que precisa ser feita de maneira independente (por exemplo atravēs do cálculo de autovalores subdominantes pertencentes a setores com $s \underline{i}$ metria bem definida). Além disso, usando condições de contorno alternativas é possivel determinar outras grandezas de interesse, co . mo por exemplo o expoente da função de correlação para pontos na superfície de um sistema semi-infinito, a dimensão dos para-fér mions, etc... E nesse sentido que o estudo numérico do espectro de cadeias finitas encontra espaço dentro desse ambicioso programa de classificação dos modelos bidimensionais.

Nesse trabalho nós investigaremos, no contexto hamilto niano ${ }^{13-15}$, o modelo de Blume-Capel ${ }^{16,17}$ com o objetivo de identi- 
ficar os operadores com suas respectivas dimensões. Existem pelo menos duas razões para estudar esse modelo. A primeira é que ele não é um modelo exatamente solúvel (pelo menos atē hoje) e a segun da é que ele é o modelo mais simples a exibir supersimetria (simetria entre bōsons e férmions). A dissertação estā esquematizada da seguinte forma: no Capitulo II são apresentadas as predições da in variância conforme para o espectro de cadeias e uma introdução à ālgebra de Virasoro. Ainda nesse capitulo verificamos essas prediÇões em um caso exatamente solúvel: o modelo de Ising em campotrans verso $^{19}$. No Capitulo III introduzimos o modelo de Blume-Emery Griffiths cuja versão mais simples, a de Blume-Capel é o tema desse trabalho. Determina-se a carga central c. no ponto tricrítico (que divide a linha de transição em duas partes, uma onde a transi

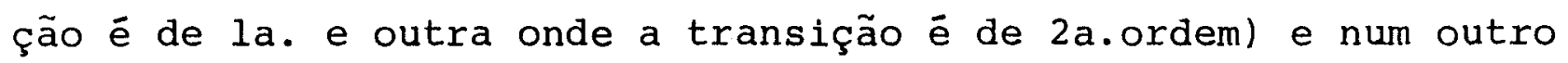
ponto da curva crítica onde se espera um comportamento do tipo -Ising. Finalmente no Capitulo. IV fazemos um estudo do efeito das condições de contorno sobre o espectro do modelo no selu ponto tricrítico. Os resultados "experimentais" são comparados com as predi ções da invartância conforme. Destacamos a determinação do expoente $\left(\eta_{s}\right)$ de superfỉcie e da dimensão do férmion ( $)$ da teoria. 
CAPITULO II

INVARIÂNCIA CONFORME EM 2 DIMENSÕES

\section{INVARIÂNCIA CONFORME}

o princípio da invariância de escala num ponto crítico tem sido bastante utilizado para extrair informações relacionadas com o comportamento crítico de sistemas estatísticos. Sabemos no entanto, desde o pioneiro trabalho de Polyakov ${ }^{1}$, que na criticalidade um sistema com interações de curto alcance e invariância trans lacional exibe uma simetria ainda maior, denominada invariância con forme, que è uma generalização da transformação de escala na qual - fator depende continuamente da posição. Esse fato porēm não se mostrou, naquela altura, de grande relevância para o estudo dos fe nômenos criticos visto que os únicos resultados que dele se obtive ram foram as regras de seleção para funções de correlação entre operadores diferentes e a dependência formal da função de três pontos em termos das dimensões anômalas dos operadores. Só recentemen te (1984) é que Belavin, Polyakov e Zamolodchikov ${ }^{8}$ mostraram o quan to a invariância conforme é poderosa na análise do comportamento crítico de sistemas bidimensionais* ${ }^{*}$.

Um exemplo simples e interessante nesse caso é a função logaritmo, que mapeia o plano inteiro-z na superficie de um cilindro. A função de correlação, em uma e outra geometria, segundo Polyakov, não são independentes e estão relacionadas pela equação

* Esta é uma consequência do grupo conforme ser infinito em duas dí mensões, jā que, nesse caso, toda função analítica $W(z)$ corres ponde a uma transformação conforme. 


$$
\left\langle\phi\left(z_{1}, \bar{z}_{1}\right) \phi\left(z_{2}, \bar{z}_{2}\right)\right\rangle=\left|w^{\prime}\left(z_{1}\right)\right|^{x}\left|w^{\prime}\left(z_{2}\right)\right|^{x}\left\langle\phi\left(w_{1}, \bar{w}_{1}\right) \phi\left(w_{2}, \bar{w}_{2}\right)\right\rangle, \text { (II. I) }
$$

onde a função de correlação da esquerda é aquela do plano infinito que na temperatura critica vale

$$
\left\langle\phi\left(z_{1}, \bar{z}_{1}\right) \phi\left(z_{2}, \bar{z}_{2}\right)\right\rangle \sim \cdot \frac{1}{\left|z_{1}-z_{2}\right|^{2 x}}
$$

e a da direita é calculada no cilindro $(x$ é a dimensão anômala do operador $\phi$ ).

\section{Escolhendo}

$$
w=\frac{L}{2 \pi} \ln z \quad z=\exp (y+i \theta)
$$

- plano-z será mapeado na cinta $-\frac{L}{2} \leqslant 1 m w \leqslant \frac{L}{2}$

Substituindo a forma da função de correlação no sistema infinito (II.2) na equação (II.1) obtemos a forma da função de cor relação na cinta

$$
\begin{aligned}
\left\langle\phi\left(w_{1}, \bar{w}_{1}\right) \phi\left(w_{2}, \bar{w}_{2}\right)\right\rangle_{T_{c}} & =\left|\omega^{\prime}\left(z_{1}\right)\right|^{-x}\left|\omega^{\prime}\left(z_{2}\right)\right|^{-x}\left|z_{1}-z_{2}\right|^{-2 x} \\
& =\left|z_{1}\right|^{x}\left|z_{2}\right|^{x}\left|z_{1}-z_{2}\right|^{-2 x} \\
& =\frac{e^{\frac{\pi}{L} \omega_{1} x} e^{\frac{\pi}{L} \bar{w}_{1} x} e^{\frac{\pi}{L} \omega_{2} x} e^{\frac{\pi}{L} \bar{w}_{2} x}}{\mid\left(e^{\frac{2 \pi}{L} \omega_{1}}-e^{\frac{2 \pi}{L} \omega_{2}}\right)\left(e^{\frac{2 \pi}{L} \bar{\omega}_{1}}-\left.e^{\frac{2 \pi}{L} \bar{\omega}_{2}}\right|^{x}\right.},
\end{aligned}
$$

ou

$$
\begin{aligned}
& \left\langle\phi\left(\omega_{1}, \bar{\omega}_{1}\right) \phi\left(\omega_{2}, \bar{w}_{2}\right)\right\rangle_{T_{C}}=\frac{(\pi / L)^{2 x}}{\left|2 \operatorname{senh} \frac{\pi}{L}\left(\omega_{1}-\omega_{2}\right)\right|^{2 x}} \\
& \text { que para }\left|\omega_{1}-\omega_{2}\right| \gg \frac{L}{\pi} \quad \text { resulta em }
\end{aligned}
$$




$$
\left\langle\phi\left(w_{1}, \bar{w}_{1}\right) \phi\left(w_{2}, \bar{w}_{2}\right)\right\rangle \sim \exp \left(-\frac{2 \pi}{L} x\left|w_{1}-w_{2}\right|\right) \text {. }
$$

Comparando esse resultado com o decaimento

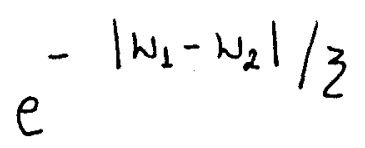

compativel com a unidimensionalidade da cinta, concluimos que

$$
L Z_{L}^{-1}=2 \pi x
$$

ou seja, a razão $\left(L / Z_{L}\right)$ é universal e está diretamente ligada ao indice crítico $\chi$.

Se estivermos trabalhando com a função de correlação spin-spin obtemos o expoente crítico $\eta$ da magnetização (já que nes se caso $2 x=\eta$ ), mas se a função de correlação envolver outros opera dores, então outros indices críticos serão obtidos.

Como a cada operador (cujo Indice crítico vale $\chi$ ) estā associado um comprimento de correlação diferente, a relação (II.8) permite determinar os indices criticos de um modelo a partir do co nhecimento dos vārios comprimentos de correlação. Estes, por sua vez, podem ser obtidos da diagonalização da matriz de transferên cia, jā que

$$
z_{2}^{i}=\left[\ln \frac{\lambda_{0}}{\lambda_{i}}\right]^{-1}
$$

\footnotetext{
*Essa relação entre os expoentes críticos e a amplitude do inverso do comprimento de correlação no ponto crítico de sistemas bidimen sionais já havia sido conjecturada em um trabalho de Derrida e de sèze ${ }^{3}$ de 1982 .
} 
onde $\lambda_{0}$ é o maior autovalor da matriz de transferência e $\lambda_{i}$ um dos autovalores seguintes, escolhido de acordo com a simetria do opera dor cuja correlação está sendo calculada.

\section{ESPECTRO}

Depois do sucesso da combinação invariância conformetfinite-size scaling, obtido atravês da relação (II.8), em determinar indices críticos, muitas outras propriedades da matriz de transferência de um sistema crítico numa cinta finita têm sido relacionadas a quantidades do sistema infinito usando-se o princípio da invariância conforme.

Para ilustrar esse novo enfoque retornamos ao cálculo da função de correlação numa cinta de largura L, com condições de con torno periódicas, calculada na formulação da matriz de transferência.

Por conveniência, escrevemos a matriz de transferência T na forma

$$
\hat{\Gamma}=e^{-a \hat{H}}
$$

onde a é o espaçamento da rede. No limite do contínuo, a $\rightarrow 0$ numa di reção (direção temporall, $\hat{\mathrm{H}}$ pode ser pensado como um operador de $\underline{\text { e }}$ volução temporal no espaço euclidiano e, identiflcado com o hamiltoniano da teoria quântica de campos em $(1+1)$ dimensões. (usaremos $w$ para denotar um ponto da cinta com $w=u+i v$, onde $u$ é a coordenada na direção temporal e $V$, na direção finita da cinta)

Nesta formulação os campos de escala $\phi(u, v)$ tornam-se operadores $\hat{\phi}(V)$ atuando no mesmo espaço de Hilbert que a matriz de transferência, e devido à invariância translacional em $v$, os auto 
estados de $\hat{\mathrm{H}}$ podem ser escolhidos como sendo os autoestados do ope rador momentum $(-i \partial / \partial v)$ com autovalores $k$ quantizados em unida des de $\frac{2 \pi}{h}$.

Introduzimos, assim, um conjunto completo de autoestados de $\hat{\mathrm{H}},|\mathrm{h}, \mathrm{k}\rangle$ com energia $E_{h}$ e momentum $\mathrm{K}$.

$$
\hat{H}|h, k\rangle=E_{n}|h, k\rangle
$$

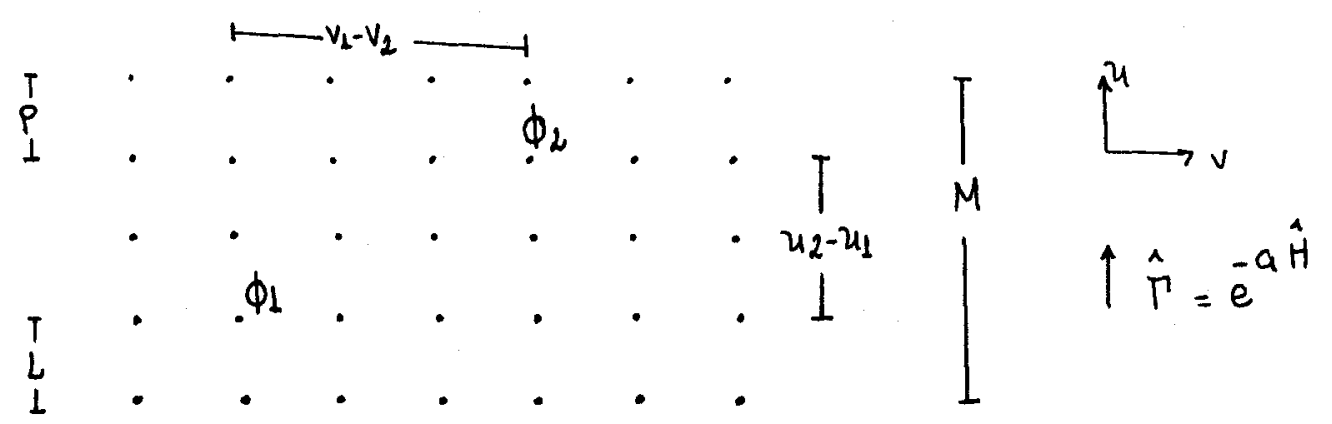

Figura II.1. Reticulado usado no cālculo da função de correlarão.

Para calcular a função de correlação entre os operadores $\hat{\phi}\left(u_{1} v_{1}\right)$ e $\hat{\phi}\left(u_{2}, v_{2}\right)$ precisamos calcular o seguinte traço

$$
\left\langle\hat{\phi}\left(u_{1}, v_{1}\right) \hat{\phi}\left(u_{2}, v_{2}\right)\right\rangle=T_{R}\left\{\hat{T}^{p} \hat{\phi}\left(v_{1}\right) \hat{\Gamma}^{\left(u_{1}-u_{2}\right)} \hat{\phi}\left(v_{2}\right) \hat{\Gamma}^{L}\right\} z^{-1}
$$

onde $Z$ é a função de partição dada por $Z=T_{R}\left\{\hat{T}^{M}\right\}$ Inserindo um conjunto completo de estados $\{|m\rangle\}$ teremos

$$
\begin{aligned}
\left\langle\phi\left(u_{1}, v_{1}\right) \phi\left(u_{2,} v_{2}\right)\right\rangle & =\sum_{m}\left\langle m\left|\hat{T}^{p} \hat{\phi}\left(v_{1}\right) \hat{\Gamma}^{\left(u_{1}\left(v_{2}\right)\right.} \hat{\phi}\left(v_{2}\right) \hat{T}^{L}\right| m\right\rangle z^{-1} \\
& =\sum_{n, m} e^{-E_{m}(P+L)} e^{-\left|u_{1}-u_{2}\right| E n_{n}}\left\langle m\left|\hat{\phi}\left(v_{1}\right)\right| n, k\right\rangle\left\langle n, k\left|\hat{\phi}\left(v_{2}\right)\right| m\right\rangle z^{-1}
\end{aligned}
$$

Como $P+\left(u_{1} \cdot u_{2}\right)+L=M$ podemos tomar $M \rightarrow \infty$, concomitantemente com $P \rightarrow \infty$ e $L \rightarrow \infty$ e $\left|u_{1}-u_{2}\right|$ fixo. Feito isso, e admitindo a unicida de do estado fundamental a equação (II.13) pode ser escrita como 


$$
\left\langle\phi\left(u_{1}, v_{1}\right) \phi\left(u_{2}, v_{2}\right)\right\rangle=\sum_{n}\left\langle 0\left|\hat{\phi}\left(v_{1}\right)\right| n_{1} k\right\rangle e^{-\left(E_{n}-E_{0} \mid\left(u_{1}-u_{2}\right)\right.}\left\langle n, k\left|\hat{\phi}\left(v_{2}\right)\right| 0\right\rangle
$$

onde os elementos de matriz dependem de $v_{L}$ e $v_{2}$ como $e^{i k\left(v_{1}-v_{2}\right)}$

Voltamos, agora, à forma da função de correlação na cinta, obtida do mapeamento conforme do plano-z com a função logarítmo:

$$
\left\langle\phi\left(w_{1}, \bar{w}_{1}\right) \phi\left(w_{2}, \bar{w}_{2}\right)\right\rangle_{T_{c}}=\frac{(\pi / L)^{2 x}}{\left|2 \operatorname{senh} \frac{\pi}{L}\left(w_{1}-w_{2}\right)\right|^{2 x}}
$$

Usando a notação $w=u+i v$, onde $u$ mede as distâncias ao longo da cinta, podemos reescrever essa expressão na forma

e, expandindo o denominador, obtemos, para $u_{1}>u_{2}$

$$
\left\langle\phi\left(u_{1}, v_{1}\right) \phi\left(u_{2}, v_{2}\right)\right\rangle=\frac{(2 \pi / L)^{x}}{\left|2 \cosh \left[\frac{2 \pi}{L}\left(u_{1}-u_{2}\right)\right]-2 \cos \left[\frac{2 \pi}{L}\left(v_{1}-v_{2}\right)\right]\right|^{x}}
$$

$$
\begin{aligned}
& \left\langle\phi\left(u_{1}, v_{1}\right) \phi\left(u_{1}, v_{2}\right)\right\rangle= \\
& =\left(\frac{2 \pi}{L}\right)^{2 x} \sum_{m_{1}=0} a_{m} a_{\bar{m}} e^{-\frac{2 \pi}{L}\left(x_{1}+m+\bar{m}\right)\left(u_{1}-u_{2}\right)} e^{\frac{2 \pi i(m-\bar{m})\left(v_{1}-v_{2}\right)}{L}}
\end{aligned}
$$

onde os coeficientes $a_{m}$ são dados por

$$
a_{m}=\frac{\Gamma(x+m)}{m ! \Gamma(x)}
$$

Comparando a expressão (II.17) com a função de correla ção obtida na formulação de matriz de transferência (II.15) vemos que a cada operador $\phi_{i}$ corresponde um número infinito de autoestados de $\hat{H}$, caracterizados pelos inteiros $(m, \bar{m})$, com energia $E_{0}+$ $+\frac{2 \pi}{L}\left(x_{i}+m+\bar{m}\right)$ e momentum $\frac{2 \pi}{L}(m-\bar{m})$. se $\phi_{i}$ possuir spin $s_{i}$ diferente de zero o momentum será modificado para $\frac{2 \pi}{L}\left(s_{i}+m-\bar{m}\right)$. 
E claro, que numa cinta cuja largura seja um número fini to de espaçamentos da rede, a matriz de transferência terá somente um nūmero finito de autoestados. Os estados excitados $\left(E_{m, \bar{m}}=\right.$ $\left.=E_{0}+\frac{2 \pi}{l}\left(x_{i}+m+\bar{m}\right), m \neq 0 e / 0 u \bar{m} \neq 0\right)$ de $\hat{H}$ são associados a operadores com dimensão de escala $x+m+\vec{m}$. (ver seção 4)

Assim, o espectro de $\hat{H}$ é constituído de sequências de ní veis de energia, "cascatas", da forma

$$
E_{0}, E_{0}+\frac{2 \pi}{L} x_{i}, E_{0}+\frac{2 \pi}{L}\left(x_{i}+1\right), \ldots, E_{0}+\frac{2 \pi}{L}\left(x_{i}+n\right), \ldots
$$

os operadores $\phi_{1}$ que correspondem aos nifveis de mais bai xa energia ( $\left.E_{0}+\frac{2 \pi}{L} x_{i}\right)$ de cada sequência $i$, ou seja, os primeiros de cada "cascata", são chamados operadores primārios. Então, conhe cendo as dimensões de escala $\chi_{i}$ dos operadores primários, podemos, através de (II.19), construir todo o espectro de $\hat{H}$.

E importante observar que todos os "gaps", $E_{n}-E_{0}$ (ver (II.19) escalam $\operatorname{com~L~}^{-1}$ e que, portanto, desaparecerão no limite $\mathrm{L} \rightarrow \infty$. A inexistência de "gaps" no ponto crítico é a causa da inva riāncia conforme (e, consequentemente, da invariância de escala) do sistema em estudo.

\section{OPERADORES PRIMARIOS}

Recentemente. Blote et al and Affleck ${ }^{12}$ mostraram que as correções devido ao tamanho finito do sistema ao autovalor mais baixo $\left(E_{0}\right)$ de $\hat{H}$ estão relacionadas a um número $\underline{c}$, chamado anomalia conforme, que além de desempenhar um papel central na análise da invariância conforme, pode ser usado para caracterizar diferentes classes de universalidade. No caso de condições de contorno periódicas esta relação se escreve como 


$$
\text { Eo } N f L-\frac{\pi C}{6 L},
$$

onde $f$ é o limite termodinâmico da energia do estado fundamental por sitio.

Belavin, Polyakov and Zamolodchikov mostraram que, se nós parametrizamos c por

$$
c=1-\frac{6}{m(m+1)},
$$

com m racional, o grupo de operadores primärios ē finito e suas di mensões de escala $(h, \bar{h})$, onde $X=h+\bar{h}$, são dados pela förmula de



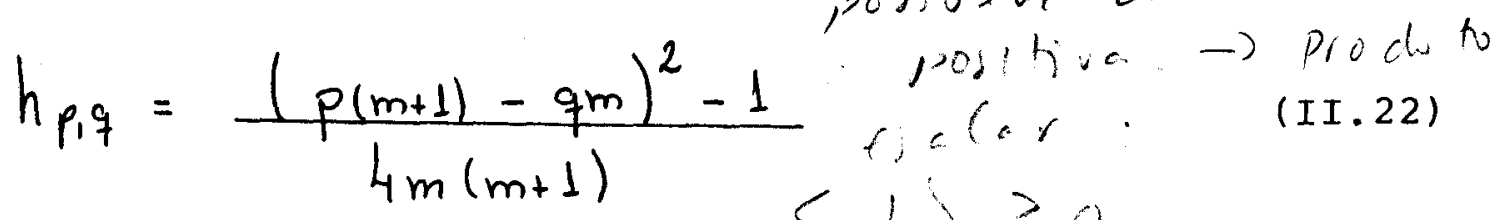

Friedan, Qiu e Shenker ${ }^{10}$ mostraram que, numa teoria unitāria com $c<1, m$ deve ser um inteiro maior que dois $(m>2)$ e, que as únicas dimensões de escala possiveis para os operadores primá rios são dados pela fórmula de Kac com $1 \leqslant q \leqslant p \leqslant m-1$. Notamos que o número de anomalia conforme $c$, realmente caracteriza uma classe de universalidade pois, tendo o valor de c obtemos um conjunto de valores de expoentes criticos através da. fórmula de Kac. Consideremos, por exemplo, os casos $c=1 / 2, c=7 / 10$ e $c=8 / 10$.

Colocando o valor $c=1 / 2$ na relação (II.21) tiramos o valor $m=3$ e, substituindo esse valor na fórmula de Kac (II.22), os seguintes valores de $h_{p} q$ são obtidos:

$$
\begin{aligned}
& h_{2,2}=1 / 16 \\
& h_{1,1}=0 \quad h_{2,1}=1 / 2
\end{aligned}
$$


e, como para condições de contorno periódicas ${ }^{*}$ somente operadores com spin $(s=h-\bar{h})$ inteiro podem ocorrer, obtemos, nesse caso, dois operadores primārios não triviais, cujas dimensões de escala são

$$
x_{1}=h_{2,2}+\bar{h}_{2,2}=1 / 8
$$

e

$$
x_{2}=h_{2,1}+\bar{h}_{2,1}=1
$$

Comparando essas dimensões com aquelas do modelo de Ising, vemos que a dimensão $X_{1}$ corresponde ao expoente crItico da magneti $z$ ação e que a dimensão $x_{2}$ está relaqionada à função de corfelação energia-energia deste modelo.

No caso $c=7 / 10$, o valor de $m$ obtido é $m=4$ e os valores de $h_{p, q}$ possiveis são:

$$
\begin{array}{ll}
h_{2,2}=3 / 80 & h_{3,3}=1 / 10 \\
h_{1,1}=0 & h_{3,2}=3 / 5 \\
h_{2,1}=7 / 16 & h_{3,1}=3 / 2
\end{array}
$$

Combinando esses valores obtemos as seguintes dimensões $(x=h+\bar{h}):$

$$
\begin{array}{ll}
x_{1}=h_{3,3}+\bar{h}_{3,3}=1 / 5 & x_{4}=h_{2,2}+\bar{h}_{2,2}=3 / 40 \\
x_{2}=h_{3,2}+\bar{h}_{3,2}=6 / 5 & x_{5}=h_{2,1}+\bar{h}_{2,1}=7 / 8 \\
x_{3}=h_{3,1}+\bar{h}_{3,1}=3 &
\end{array}
$$

Estas dimensões obtidas são exatamente as dimensões dê escala do modelo de Ising tricrítico, conjecturadas por Nienhuis 20 .

\footnotetext{
* No caso de condições periódicas de contorno o grupo de simetria é $G=\Gamma \otimes \bar{\Gamma}$ onde $\bar{\Gamma}(\bar{\Gamma})$ è o grupo das substituições analiticas da va riável $Z(\bar{z})$, e por essa razão as dimensões das representações são somas de dois h's.
} 
No caso $c=8 / 10 \quad(m=5)$ a fórmula de Kac fornece o seguinte conjunto de dimensöes:

$\begin{array}{rlrl} & & \mathrm{h}_{4,4}=1 / 8 \\ \mathrm{~h}_{3,3}=1 / 15 & \mathrm{~h}_{4,3}=2 / 3 \\ \mathrm{~h}_{2,2}=1 / 40 & \mathrm{~h}_{3,2}=21 / 40 & \mathrm{~h}_{4,2}=13 / 8 \\ \mathrm{~h}_{1,1}=0 & \mathrm{~h}_{2,1}=2 / 5 & \mathrm{~h}_{3,1}=7 / 5 & \mathrm{~h}_{4,1}=3\end{array}$

Como sempre acontece nesses casos, o conhecimento do conjunto $\left\{h_{p}, q\right\}$ não é suficiente para determinar o modelo que se está descrevendo e muito menos para estabelecer a correta conexão entre os expoentes encontrados e os correspondentes operadores. Para re solver essa questão Gehlen e Rittenberg ${ }^{21}$ estudaram o modelo de Potts com 3 estados por sitio, sujeito a uma variedade de condições de contorno e encontraram explicaçöes para um subconjunto das repre sentações (II.27) associadas ao $c=8 / 10$. Como esse valor de $\underline{c}$ deve descrever também o modelo de Ising tetracrítico parecia razoāvel não esgotar, com o Potts-3, todo o conjunto dos h's. Entretanto, con forme apontado recentemente por $\operatorname{Cardy}^{22}$, é possivel encontrar as di mensõès ausentes da anālise de Gehlen e Rittenberg considerando um novo tipo de condição de contorno que chamaremos de "conjugada".

\section{INVARIÂNCIA CONFORME E A ALLGEBRA DE VIRASORO}

Sistemas estatísticos em duas dimensões podem ser inter pretados como sistemas quânticos unidimensionais, segundo a conhecí da técnica da matriz de transferência. Assim, no ponto crítico o comportamento do sistema pode ser descrito, muito adequadamente , por operadores da teoria quântica de campos. Uma característica im" $\rightarrow$ pos, , s pfor citiw

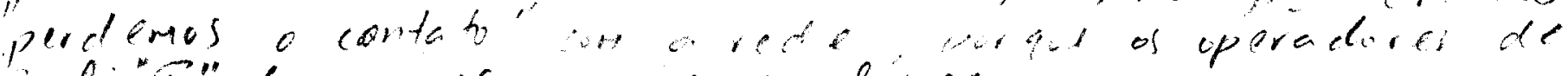




portante da teoria de campos é que os estados têm a norma positiva, isto é, o espaço de estados é um espaço de Hilbert e, portanto, a teoria é unitária. Friedan, Qiu e Shenker ${ }^{10}$ têm estudado o papel desta propriedade nas teorias de campos conforme.

No estuco do grupo de transformações conforme nos deparamos com o tensor momento-energia $\mathrm{T}_{\mu \nu}$. Em teorias quânticas de campos, o tensor momento-energia é o operador que gera transformações locais nas coordenadas. Ele é o operador que modifica localmente o sistema, enfraquecendo acoplamentos numa direção e fortalecendo na outra. Em duas dimensōes, o tensor momento-energia tem quatro compo nentes e, se existe invariância de escala global?, é simétrico e com traço nulo, resultando apenas duas componentes independentes. Da lei de conservação $\partial_{\mu} \mathrm{T}_{\mu \nu}=0$, as duas combinações $\mathrm{T}=\mathrm{T}_{11}+i \mathrm{~T}_{12} \mathrm{e}$ $\overline{\mathrm{T}}=\mathrm{T}_{11}-i \mathrm{~T}_{12}$ são independentes $\mathrm{e}$ só dependem das combinações $\mathrm{Z}=$ $=x+i y$ e $\bar{z}=x-i y$.

Com o objetivo de identificar os operadores que geram trans lações, rotações e dilatações no plano, expandimos T e $\bar{T}$ nas suas componentes de Fourier:

$$
T(z)=\sum_{n=-\infty}^{\infty} L_{n} z^{-n-2} \quad \bar{T}(\bar{z})=\sum_{n=-\infty}^{\infty} \bar{L}_{n} \bar{z}^{-n-2}
$$

e, em seguida examinamos o efeito de uma transformação conforme infinitesimal.

$$
z \rightarrow z+\varepsilon(z) \quad, \quad \bar{z} \rightarrow \bar{z}+\varepsilon(\bar{z})
$$

onde

$$
E(z)=\sum_{n=-\infty}^{\infty} \ln z^{n}, \quad \bar{\varepsilon}(\bar{z})=\sum_{n=-\infty}^{\infty} \bar{\varepsilon}_{n} \bar{z}^{n}
$$


por exemplo, geram as dilatações em $\mathrm{z}$ e $\overline{\mathrm{Z}}$, respectivamente:

$$
\begin{aligned}
& z \rightarrow z+\mathcal{E} \quad, \quad \bar{z} \rightarrow \bar{z}+\bar{\varepsilon} \bar{z} \quad \text { (II.36) } \\
& \text { o operador } \mathrm{L}_{\mathrm{o}}+\overline{\mathrm{L}}_{\circ} \text { gera a dilatação do plano bidimensional } \\
& z \rightarrow z+\lambda z \quad \bar{z} \rightarrow \bar{z}+\lambda \bar{z}, \quad, \lambda \text { é um nủmero real (II.37) }
\end{aligned}
$$

Similarmente, podemos ver que o operador $\mathrm{L}_{\mathrm{O}}-\overline{\mathrm{L}}_{\mathrm{O}}$ gera rota ções e os operadores $L_{-1}$ e $\overline{\mathrm{L}}_{-1}$ geram translações. Os operadores $\mathrm{L}_{0^{\prime}}$ $\overline{\mathrm{L}}_{0^{\prime}} \mathrm{L}_{-1}, \overline{\mathrm{L}}_{-1}$ juntamente com $\mathrm{L}_{1}$ e $\overline{\mathrm{L}}_{1}$ formam, entäo, o grupo de trans formações conforme globais. E possível mostrar ainda que de modo ge ral, os operadores $L_{n}$ e $\bar{L}_{n}$ satisfazem as seguintes relações de comu tação e hermiticidade:

$$
\begin{aligned}
& L_{n}^{+}=L_{-n}, \quad \bar{L}_{n}^{+}=\bar{L}_{-n} \\
& {\left[L_{m}, L_{n}\right]=(m-n) L_{m+n}+\frac{1}{12} c m\left(m^{2}-1\right) \delta_{m+n, 0}} \\
& {\left[\bar{L}_{m}, \bar{L}_{n}\right]=(m-n) \bar{L}_{m+n}+\frac{1}{12} C m\left(m^{2}-1\right) \delta_{m+n, 0}} \\
& {\left[L_{m}, \bar{L}_{n}\right]=0}
\end{aligned}
$$

onde o número adimensional $\underline{c}$ é a carga central ou númeró de anoma lia conforme.

A álgebra formada pelos $L_{n}\left(\bar{L}_{n}\right)$ é a chamada álgebra de Virasoro, portanto, a ālgebra das transformações conforme locais é um rasoro, portanto, a algebra das transforkço produto de duas álgebras de virasoro, que comutam entre si. A álgebra de Virasoro é a extensão central da álgebra dos operadores dife rencials 


$$
l_{n}=z^{n+1} \frac{d}{d z} \quad n=0, \pm 1, \pm 2, \ldots
$$

As regras de comutação para esses operadores são facilmen te obtidas como mostramos abaixo:

$$
\begin{aligned}
& \ln \operatorname{lm} \phi-\operatorname{lm} \ln \phi=\ln z^{m+1} \frac{d}{d z} \phi-\ln z^{n+1} \frac{d}{d z} \phi= \\
& =(m+1) z^{n+1} z^{m} \frac{d \phi}{d z}+z^{n+1} z^{m+1} \frac{d^{2} \phi}{d z^{2}}-(n+1) z^{m+1} z^{n} \frac{d \phi}{d z}-z^{m+1} z^{n+1} \frac{d \phi}{d z^{2}} \\
& \ln \operatorname{lm} \phi-\operatorname{lm} \cdot \ln \phi=(m-n) z^{n+m+1} \frac{d \phi}{d z}=(m-n) \ln +m \phi
\end{aligned}
$$

e, então;

$$
\left[l_{n} l_{m}\right]=(m-n) l_{n+m}
$$

Voltando a álgebra de Virasoro e escolhendo novas coordenadas $\sigma$ e $\tau$, definidas por

$$
Z=\exp (\tau+i \sigma) \quad, \quad \xi=\exp (\tau-i \sigma) .
$$

obtemos que o Hamiltoniano pode ser escrito como

$$
H=L_{0}+\bar{L}_{0}
$$

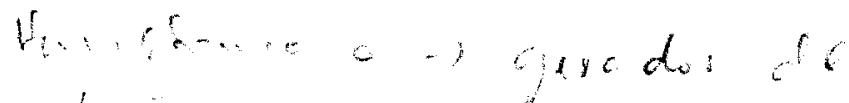

$$
\begin{aligned}
& \text { traissing (II.42) }
\end{aligned}
$$

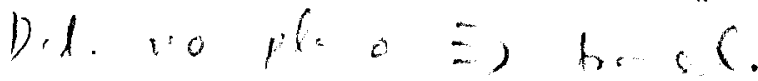

isto é, os autoestados de $\mathrm{H}$ são autoestados de $L_{0}$ e $\overline{\mathrm{L}}_{\mathrm{O}}$. Das rela ções de comutação (II.38) com $n=0$ vemos que

$$
\left[L_{0}, L_{n}\right]=+n L_{n}
$$


e, trabalhando com autoestados $|h\rangle$ de $L_{0}$ cujos autovalores são deno tados por $h$, teremos

$$
\begin{aligned}
& \operatorname{loln}|h\rangle-\ln L_{0}|h\rangle=-n \ln |h\rangle, \\
& \operatorname{LoL}|h\rangle-h \ln |h\rangle=-n \ln |h\rangle, \\
& L_{0}(\operatorname{Ln}|h\rangle)=\left(h_{-n}\right)(\operatorname{Ln}|h\rangle),
\end{aligned}
$$

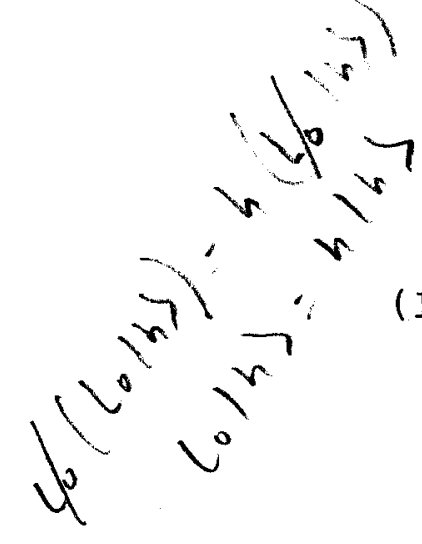

- que possibilita a identificação de $L_{n}$ com um operador de levantamente se $n<0$ e, como um operador de abaixamento se $n>0$.

Assim, o estado $|h\rangle$ é um estado "fundamental" se ele for aniquilado por todos os operadores de abaixamento, isto é,

$$
L_{0}|h\rangle=h|h\rangle, \quad L_{h}|h\rangle=0 \quad n>0
$$

o espaço de Hilbert é gerado aplicando o operador de le vantamento no estado fundamental $|0\rangle$. Ałém disso, o estado fundameṇ tal $|0\rangle$ deve ser aniquilado por $L_{h^{\prime}} \bar{L}_{n}$ se $n \geqslant-1$, isto $\bar{e}$

$$
l_{n}|0\rangle=I_{n}|0\rangle=0 \quad n \geqslant-1
$$

para que o tensor momento-energia seja não-singular em $z=0$, jā que

$$
\Gamma(z)=\sum_{n=-\infty}^{\infty} \operatorname{Ln} \bar{z}^{-n-2} \quad \bar{T}(\bar{z})=\sum_{n=-\infty}^{\infty} \bar{L}_{n} \bar{z}^{(-n-2)}
$$

o significado físico de $\mathrm{h}$ e $\overline{\mathrm{h}}$ pode ser entendido estudando as funções de correlação dos campos que são invariantes frente a transformações conforme globais (geradas pelos $L_{n}, \bar{L}_{n}$ para $n=-1,0,1$ ). Em particular, pode-se determinar a forma da função de dois pontos: 


$$
\begin{aligned}
\frac{e^{,(\Delta . j)}}{\mid r 1^{2(\theta+j)}} & 20 \\
\langle 0|\phi(z, z) \phi(0,0)| 0\rangle & =\left\langle 0\left|\phi\left(r e^{i \theta}, r e^{-i \theta}\right) \phi(0,0)\right| 0\right\rangle \\
& =r^{-2(h+h)} e^{2 i(h-\bar{h})}
\end{aligned}
$$

vemos, então, que $\phi(z, \bar{z})$ tem dimensão $x=h+\bar{h}$ e spin $s=h-\bar{h}$.

A definição dos operadores primārios pode ser introduzida neste contexto da expansão de $T(z)$ com o operador $\phi\left(Z^{\prime}, \bar{z}^{\prime}\right)$

$$
T(z) \phi\left(z^{\prime}, \bar{z}^{\prime}\right)=\sum_{n=-\infty}^{\infty}\left(z-z^{\prime}\right)^{-2-n} L_{n} \phi\left(\bar{z}^{\prime}, \bar{z}^{\prime}\right)
$$

que leva à seguinte relação de comutação

$$
\left[L_{n}, \phi(z, \bar{z})\right]=z^{n+1} \partial z \phi(z, \bar{z})+h(n+1) z^{n} \phi(z, \bar{z}) \cdot \text { (II.49) }
$$

Portanto, se $\phi$ tiver dimensão de escala $h$, os operadores $L_{n}$ terão dimensão $x=h-h$. Como numa teoria unitária os operadores não podem ter dimensão negativa $(x<0)$, existirão operadores $\phi$ para os quais

$$
\ln \phi=0 \quad, \quad n>0
$$

que serão chamados operadores primärios. Os operadores $L_{n} \phi(n<0)$ não têm essa propriedade. Estes operadores juntamente com o operador . primário $\phi$ constituem um bloco conforme, cujos primeiros membros são:

nĩve1

$\phi$

$$
L_{-1} \phi
$$

$\mathrm{L}_{-2} \phi, \mathrm{L}_{-1}^{2} \phi$

$L_{-3} \phi, L_{-1} L_{-2} \phi, L_{-1}^{3} \phi$ 
O número de operadores no nível $\mathrm{N}$ é $\mathrm{P}(\mathrm{N})$ ), o número de partições do intelro $\mathrm{N}$ e todos eles têm dimensão de escala $\mathrm{x}+\mathrm{N}$.

E importante notar que nem todos os estados obtidos pela aplicação de operadores do mesmo nîvel são independentes e como con sequência disso a degenerescência do nivel $\mathbf{N}$ não è, em geral, igual a $P(N)$. Para calcular corretamente a degenerescência de um nível é preciso usar a tabela de caracteres correspondente que pode ser obtida através da fórmula calculada por Rocha-Carı́di (veja Apêndice A e Capitulo IV).

Como a variação de um operador secundário (que não é primáriol pertencente a um dado bloco conforme sob uma transformação conforme infinitesimal pode ser expressa linearmente em termos das representações deste mesmo bloco conforme, podemos identiflcar cada bloco conforme com uma representação Irredutivel da ālgebra do grupo conforme. O espaço de Hilbert da teoria conforme pode ser, então, decomposto em representações irredutíveis das duas ālgebras de Vira soro formadas pelos operadores $L_{n}$ e $\bar{L}_{n}$. 
5. APLICAÇÃO AO MODELO DE ISING EM CAMPO TRANSVERSO

Nessa seção nós veriricaremos as predições da invariância conforme em um modelo exatamente solúvel que é a cadeia de L spins de Ising em um campo transverso $\lambda$ cujo hamiltoniano com condições livres de contorno è

$$
H_{F}=-\lambda \sum_{n=1}^{L} \sigma_{n}^{z}-2 \sum_{n=1}^{L-1} \sigma_{n}^{x} \sigma_{n+1}^{x}
$$

onde $G^{x}, G^{z}$ são matrizes de Pauli. Para condiçóes periōdicas e añ tiperiódicas de contorno um termo extra $H_{1}=\mp 2 G_{4}^{x} \sigma_{1}^{x}$, respectiva mente, è incluido no hamiltoniano. No limite $L \rightarrow \infty$, o estado funda mental é singular em $\lambda_{c}=1$ (a cadeia de Ising num campo transverso fol estudada em detalhes por Katsura, Pfeuty e Boccara e Sarma 1 . 0 modelo pode ser resolvido introduzindo-se os operadores de crlaçãoe aniquilação de férmions e, então, diagonalizando-se exatamente o hạ miltoniano resultante por uma transformação canönica.

Substituímos os Paulions por Férmions através de uma trans formação de Jordan-Wigner que se escreve

$$
c_{j}^{+}=\frac{1}{2}\left(\sigma_{j}^{x}+i \sigma_{j}^{y}\right) \prod_{k<j}\left[-\sigma_{k}^{z}\right],
$$

e

$$
c_{j}=\frac{1}{2} \prod_{k<j}\left[-\sigma_{k}^{z}\right]\left(\sigma_{j}^{x}-i \sigma_{j}^{y}\right),
$$

Em termos dos operadores fermiônicos $C_{n}, c_{n}^{+} \circ$ hamiltoniano $H_{F}$ é modificado para 
soluçöes da equação

$$
\lambda^{-1}=\frac{\operatorname{sen}[(l+1) k]}{\operatorname{sen}(l k)} \text {. }
$$

Em $\lambda=\lambda_{c}=1$, isso se reduz a $\operatorname{tanLk}=\operatorname{cotg}(k / 2)$, ou ainda

$$
|k|=\frac{(2 m+1)}{(2 L+1)} \pi \quad m=0,1, \ldots, L-1
$$

Para $L=4$, por exemplo

$$
|k|=\frac{\pi}{9}, \frac{3 \pi}{9}, \frac{5 \pi}{9} \text { e } \frac{7 \pi}{9} \text {. }
$$

A energia do estado fundamental corresponde ao estado sem nenhum férmion, de forma que

$$
\begin{aligned}
E_{0}(F) & =-\frac{1}{2} \sum_{m=0}^{L-1} \wedge\left(\frac{2 m+1}{2 L+1} \pi\right)= \\
& =\frac{1}{2}\left(1-\operatorname{cosec} \frac{\pi}{2(2 L+1)}\right) .
\end{aligned}
$$

o primeiro estado excitado è obtido colocando-se uma "par ticula" de momento $k=\pi /(2 \mathrm{~L}+1)$, de modo que o 18 "gap" dentro das condições livres de contorno é dado por

$$
\begin{aligned}
& G^{(-)}(F)=\Lambda\left(\frac{\pi}{(2 L+1)}\right)=2 \operatorname{sen}\left(\frac{\pi}{2 L+1}\right)_{E,(F)} \\
& G(f)=E_{1}(F)-\varepsilon_{0} t=-\frac{1}{2} \sum_{t} 1(t)+1\left(\frac{t}{t+1}+\frac{5}{2}(t)\right. \\
& \text { para calcular o segundo "gap" ê preciso excitar duas "par } \\
& \text { tículas", uma de momento } k=\pi /(2 \mathrm{~L}+1) \text { e outra de momento } k=3 \pi /(2 \mathrm{~L}+1) \text {. } \\
& \text { de forma que: }
\end{aligned}
$$




$$
\begin{aligned}
G^{(t)}(F) & =\Lambda\left(\frac{\pi}{2 L+1}\right)+\Lambda\left(\frac{3 \pi}{2 L+1}\right)= \\
& =2\left(\operatorname{sen} \frac{\pi}{2(2 L+1)}+\operatorname{sen} \frac{3 \pi}{2(2 L+1)}\right) .
\end{aligned}
$$

Para condições periódicas de contorno com $\mathcal{N}$ par ou condi-

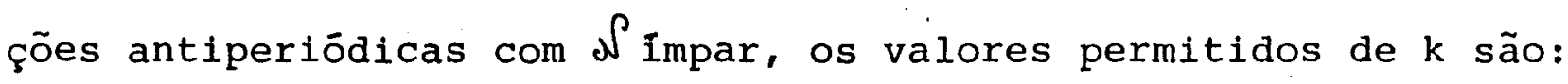

$$
k=\left[\frac{(2 m+1)}{L}\right] \pi \quad m=0,1, \ldots, L-1
$$

enquanto que para condições periōdicas com $\int$ impar ou antiperiódi -



$$
K=\left(\frac{2 m}{L}\right) \pi \quad K=\frac{\pi}{L}(k+2, \ldots, L-1
$$

Os dois grupos de k's possuem dois estados fundamentais dí ferentes que, em $\lambda=\lambda_{c}=1$ possuem $d \int$ par. Assim, as energias dos estados fundamentais para condições periódicas e antiperiōdicas de contorno, respectivamente, são dadas por

$$
\begin{aligned}
& E_{0}(P)=-\frac{1}{2} \sum_{m=0}^{L-1} \wedge\left(\frac{2 m+1}{L} \pi\right)=-\operatorname{cosec}\left(\frac{\pi}{2 L}\right) . \\
& E_{0}(A)=-\frac{1}{2} \sum_{m=0}^{L-1} \wedge\left(\frac{2 m}{L} \pi\right)=-\operatorname{cotg}\left(\frac{\pi}{L}\right) .
\end{aligned}
$$

Note que $E_{O}(A)-E_{O}(P)=\tan (\pi / 4 L)$. Alēm disso, o primeiro estado excitado no setor of par corresponde a 
26

$$
\begin{aligned}
& E_{1}^{(t)}(p)=-\frac{1}{2} \sum_{k} \Lambda(k)+\Lambda\left(\frac{\pi}{L}\right)+\Lambda\left(\frac{(2 L-1) \pi}{L}\right) \\
& \text { resultando no "gap" } \mathrm{G}^{(+)}(\mathrm{P}) \\
& G_{1}^{(t)}(p)=\Lambda\left(\frac{\pi}{L}\right)+\Lambda\left(\frac{(2 L-1) \pi}{L}\right)=4 \operatorname{sen}\left(\frac{\pi}{2 L}\right) .
\end{aligned}
$$

E possivel formar estados com $k_{\text {total }} \neq 0$, excitando, por exemplo

$$
\frac{\pi}{L}, \frac{3 \pi}{L} \Rightarrow K_{T}=\frac{\pi}{L}+\frac{3 \pi}{L}=\frac{4 \pi}{L}
$$

ou ainda

$$
\frac{\pi}{L},(2 L-3) \frac{\pi}{L} \Rightarrow K_{T}=-\frac{2 \pi}{L} .
$$

Eles terão energia mais alta, obedecendo a relação de dispersão $\mathrm{E}=$ $=\mathrm{p} c$.

Para obter o primeiro estado excitado no setor $\mathcal{N}$ ímpar , dentro das condições periódicas, excitamos uma partícula de k=0, mas com isso não custa nada em energia $(\Lambda(0)=0)$ teremos $E_{1}^{(-)}(P)=$ $=E_{0}(A)$ : Assim, o primeiro "gap" de condições periódicas de contorno dado por

$$
E_{n}^{(-)}(P)=-\frac{1}{2} \sum_{n=0}^{i=1} A\left(\frac{2 N \pi)+\lambda(10)=(1+1)}{l}\right.
$$

$$
\begin{aligned}
G_{1}^{(-)}(P) & =\Lambda(0)+E_{0}^{(t)}(A)-E_{0}^{(+)}(P)= \\
& =E_{0}^{(t)}(A)-E_{0}^{(+)}(P)=\tan \left(\frac{\pi}{4 L}\right) . \\
-\frac{1}{2} 5 & =\frac{1}{2}
\end{aligned}
$$


coincide com a diferença de energia entre o estado fundamental com condições periódicas de contorno e aquele com condiçöes antiperiōdi cas de contorno. Esse fato é compatível com a igualdade entre a dimensão dos operadores ordem e desordem, como veremos mais adiante.

o primeiro estado excitado das condições antiperiódicas de contorno ( $\mathcal{N}$ impar) será igual a $E_{O}(P)$ a menos de uma particula que aumenta a energia para

$$
E_{1}^{(-)}(A)=E_{0}(P)+\Lambda\left(\frac{\pi}{L}\right),
$$

resultando nim "gap" $\mathrm{G}^{(-)}(\mathrm{A})$ igual à

$$
G^{(-)}(A)=2 \operatorname{sen}\left(\frac{\pi}{2 L}\right)-\tan \left(\frac{\pi}{4 L}\right) .
$$

Os primeiros "gaps" para condições periōdicas e antipe riódicas de contorno, são, então, dados por:

$$
\begin{aligned}
& G^{(-)}(P)=\Lambda(0)+E_{0}(A)-E_{0}(P)=\tan \left(\frac{\pi}{4 L}\right) \\
& G^{(t)}(P)=\Lambda\left(\frac{\pi}{L}\right)+\Lambda\left[\frac{(2 L-1)}{L} \pi\right]=4 \operatorname{sen}\left(\frac{\pi}{2 L}\right) \\
& G^{(-1}(A)=\Lambda\left(\frac{\pi}{L}\right)+E_{0}(P)-E_{0}(A)= \\
& =2 \operatorname{sen}\left(\frac{\pi}{2 L}\right)-\tan \left(\frac{\pi}{4 L}\right) \\
& G^{(t)}(A)=\Lambda(0)+\Lambda\left(\frac{2 \pi}{L}\right)=2 \operatorname{sen}\left(\frac{\pi}{L}\right)
\end{aligned}
$$


que, no limite $L \rightarrow \infty$, resultarão em:

$$
\varepsilon^{(-)}=\lim _{L \rightarrow \infty} L G L_{L}^{(-)}= \begin{cases}\pi / 4 & \text { para condiçöes periódicas de contorno } \\ \pi / 2 & \text { para condições 1ivres de contorno } \\ 3 \pi / 4 & \text { para condições antiperiódicas de con- } \\ & \text { torno }\end{cases}
$$

e

$$
\begin{aligned}
\varepsilon^{(t)}=\lim _{l \rightarrow \infty} L G_{L}^{(t)}=2 \pi \quad & \text { para os três tipos de condi - } \\
& \text { ções de contorno. }
\end{aligned}
$$

Lembrando que, os "gaps" estão relacionados às dimensões de escala por

$$
\begin{aligned}
& L G=2 \pi x^{\text {(volume) }} \text { para condiçöes periódicas e antiperió- } \\
& \text { dicas } \\
& \text { (II. 78) }
\end{aligned}
$$

$$
L G=\pi x^{(\text {superff́cie })} \text { para condiçöes livres }
$$

obtemos os seguintes resultados:

$$
x_{0}^{\text {(volume) }}=1 / 8 \quad x_{6}^{\text {(juperf) }}=1 / 2
$$

onde $X_{r}$ é a dimensão da magnetização, e

$$
x_{t}^{\text {(volume) }}=1 \quad x_{\epsilon}^{\text {(superf) }}=2
$$


onde $X_{f}=(2-1 / \psi)$ é a dimensão do operador energia.

As condições antiperiódicas de contorno exigem um pouco mais de cuidada pois elas contém mais informações relevantes do que se pensa. Vejamos isso: para o modelo de Ising, a imposição de condições antiperiódicas é equivalente à inserção de dois operadores desordem $\mu$ em $u= \pm \infty$. A função de correlação de um operador $\phi$, no sistema com condições antiperiódicas de contorno, pode então ser escrita em termos de uma outra calculada em um sistema com con dições periódicas de contorno, conforme segue:

$$
\left\langle\phi(u) \phi\left(u_{2}\right)\right\rangle_{A}=\lim _{\substack{u_{1} \rightarrow-\infty \\ u_{2}^{\prime} \rightarrow+\infty}} \frac{\left\langle\mu\left(u_{1}^{\prime}\right) \phi\left(u_{1}\right) \phi\left(u_{2}\right) \mu\left(u_{2}^{\prime}\right)\right\rangle_{P}}{\left\langle\mu\left(u_{1}^{\prime}\right) \mu\left(u_{2}^{\prime}\right)\right\rangle_{P}}
$$

o comprimento de correlação é obtido tomando-se o limite $u_{2}-u_{1}$ grande, depois dos limites en $u_{1}^{\prime}$ e $u_{2}^{\prime}$. o autoestado de $\hat{H}$ que domina neste limite corresponde ao pperador com menor dimensão $X$ que é gerada na expansão de curta q̣istância de $\mu$ e $\phi$. Para o mode 10 de Ising, se $\phi=\sigma$ (o operador da magnetização), o operador mais relevante gerado ê o férmion $\psi$, que tem $\chi_{F}=1 / 2$, correspondendo ao autovalor $\mathrm{E}_{\mathrm{o}}+\pi / \mathrm{L}$. Deste, deve ser subtralda a dimensão corres pondendo a $\mu$ no denominador, que é igual à de $\sigma$ por dualidade. Assim

$$
L G^{(-)}(A)=2 \pi\left(X_{F}-X_{\mu}\right)=2 \pi\left(\frac{1}{2}-\frac{1}{8}\right)=\frac{3}{4} \pi
$$

Se consideremos os "gaps" formados com autovalores mais altos obteremos as "cascatas" (veja seção II do Capỉtulo II) associadas a essas dimensões de escala, listadas em (II.77).

Vejamos, por exemplo, o que acontece no caso de condi - 
ções periódicas de contorno. Os autovalores no setor par ( $f$ par ) são obtidos excitando-se um nümero par de "partículas" com momentos dados por:

$$
K=\frac{(2 m+1)}{L} \pi \quad m=0,1, \ldots L-1
$$

Assim, além do estado fundamental

$$
E_{0}(p)=-\frac{1}{2} \sum_{k} \Lambda(k)=-\frac{1}{2} \sum_{m=0}^{b-1} \Lambda\left(\frac{2 m+1}{L} \pi\right),
$$

e do 18 estado excitado (no setor par)

$$
E_{1}^{(t)}(p)=-\frac{1}{2} \sum_{k} \Lambda(k)+\Lambda\left(\frac{\pi}{L}\right)+\Lambda\left(\frac{(2 L-1)}{L} \pi\right)
$$

outros autovalores podem ser obtidos excitando-se, por exemplo, uma particula com $k=\pi / L$ e outra com $k=3 \pi / L$, e assim por diante. No caso mencionado a energia é

$$
E_{2}^{(t)}(p)=-\frac{1}{2} \sum_{k} \Lambda(k)+\Lambda\left(\frac{\pi}{L}\right)+\Lambda\left(\frac{3 \pi}{L}\right)
$$

cujo momento total é $K_{T}=\frac{\pi}{I}+\frac{3 \pi}{L}=\frac{4 \pi}{I}$.

A diferença de energia $\left(E_{2}^{+}-E_{0}\right)^{L}$ è dada por

$$
G_{2}^{(+)}(p)=2 \operatorname{sen}\left(\frac{\pi}{2 L}\right)+2 \operatorname{sen}\left(\frac{3 \pi}{2 L}\right),
$$

que no limite $L \rightarrow \infty$ se reduz a

$$
\lim _{L \rightarrow \infty} L G_{2}^{(t)}(p)=\pi+3 \pi=4 \pi=2 \pi x_{2}^{(t)}=2 \pi\left(x_{\epsilon+1}\right)
$$


31

$\operatorname{com} \chi_{2}^{(t)}=X_{E}+1$.

Podemos excitar uma particula com $k=\frac{5 \pi}{L}$ ao invés de $k=\frac{3 \pi}{L}$, o que resultará em:

$$
E_{3}^{(+)}(P)=-\frac{1}{2} \sum_{k} \Lambda(k)+\Lambda\left(\frac{\pi}{L}\right)+\Lambda\left(\frac{5 \pi}{L}\right)
$$

de forma que

$$
G_{3}^{(t)}(P)=2 \operatorname{sen}\left(\frac{\pi}{2 L}\right)+2 \operatorname{sen}\left(\frac{5 \pi}{L}\right)
$$

e

(II. 88)

$$
\lim _{L \rightarrow \infty} l G_{3}^{(t)}(P)=6 \pi=2 \pi X_{3}^{(t)}=2 \pi\left(x_{\epsilon}+2\right)
$$

No setor Impar ( $\mathcal{S}$ impar) os autovalores são obt1dos excitando-se um número impar de particulas com momentos dados por:

$$
K=\frac{2 m}{L} \pi \quad m=0,1, \ldots, L-1 .
$$

Dessa forma, analogamente ao setor par obtém-se:

$$
\begin{aligned}
& E_{2}^{(-)}(P)=-\frac{1}{2} \sum_{k} \Lambda(k)+\Lambda\left(\frac{2 \pi}{L}\right) \\
& G_{2}^{(-)}(P)=\tan \left(\frac{\pi}{4 L}\right)+2 \operatorname{sen} \frac{\pi}{L} \\
& \lim _{b \rightarrow \infty} L G_{2}^{(t)}(P)=\frac{\pi}{4}+2 \pi=2 \pi\left(x_{r}+1\right), \quad x_{r}=1 / 8
\end{aligned}
$$

ou ainda: 


$$
\begin{aligned}
& E_{3}^{(-)}(P)=-\frac{1}{2} \sum_{K} \wedge(K)+\Lambda(0)+\Lambda\left(\frac{2 \pi}{L}\right)+\Lambda\left(\frac{2(L-1) \pi}{L}\right) \\
& G_{3}^{(-)}(P)=\tan \left(\frac{\pi}{4 L}\right)+2 \operatorname{sen} \frac{\pi}{L}+2 \operatorname{sen}(L-1) \frac{\pi}{L}
\end{aligned}
$$

que no limite $\mathrm{L} \rightarrow \infty$ resulta em

$$
\lim _{L \rightarrow \infty} L G_{3}^{(-)}(P)=\frac{\pi}{4}+2 \pi+2 \pi=2 \pi\left(x_{r}+2\right)
$$

Dessa forma todo o espectro pode ser construído, simples mente como "cascatas" dos operadores primários. Vale a pena mencionar que isso só ocorre quando o modelo é mỉnimo, isto é, tem um con junto finito de operadores primários. Há casos, como exèmplificado pelo modelo de Ising com defeitos lineares em que o conjunto de ope radores primários é infinito tornando assim a construção do espec tro altamente não trivial. 
CAPITULO III

O MODELO DE BLUME-CAPEL

1. O MODELO

O modelo $\mathrm{BEG}^{24}$, Blume-Emery-Griffths, foi introduzido em 1971 numa tentativa de explicar qualitativamente o diagrama de fa ses da mistura $\mathrm{He}^{3}-\mathrm{He}^{4}$ (veja figura III.1) e, consiste de uma generalização do modelo de Ising para spin 1. O valor $\mathrm{Sz}=0$ corresponde aos átomos de $\mathrm{He}^{3}$ e os valores +1 e -1 aos ätomos de $\mathrm{He}^{4}$. Na sua forma mais geral, o Hamiltoniano com interações de vizinhos mais próximos pode ser escrito como

$$
\begin{aligned}
H= & -H \sum_{j=1}^{L} S_{j}+\Delta \sum_{j=1}^{L} S_{j}^{2}-J \sum_{\langle i j\rangle} S_{i} S_{j} \\
& -H_{3} \sum_{\langle i, j\rangle} S_{i} S_{j}\left(S_{i}+S_{j}\right)-K \sum_{\langle i,\rangle} S_{i}^{2} S_{j}^{2}
\end{aligned}
$$

onde cada spin $\mathrm{s}_{\mathrm{k}}$ da rede pode assumił os valores $-1,0$ e 1 . O parâmetro H representa o campo magnética, $\Delta$ é o campo cristalino, são interações de troca bilineares e biquadrăticas, respectivamente, e $\mathrm{H}_{3}$ introduz uma perturbação magnētıca de $3 \mathrm{a}$.ordem.

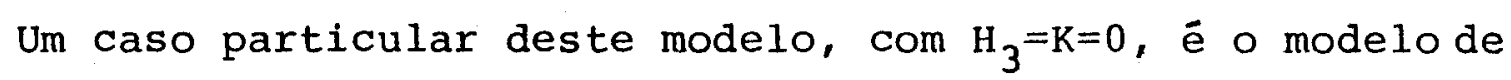
Blume-Capel, introduzido independentemente por Blume ${ }^{16}$ e Capel ${ }^{17} \mathrm{em}$ 1966. Esse modelo, embora mais simples, já apresenta um comportamen to bem mais rico que o modelo de Ising, com uma transição de fase que pode ser continua ou de la.ordem, dependendo do valor do campo cristalino $\Delta$. O diagrama de fases do modelo a campo nulo está mostrado na figura III.2, enquanto que na figura III.3 está representa 
do o diagrama para $H \neq 0$. Como se pode ver nesta última figura, o pon to tricrítico é o encontro de três linhas $\lambda$ e, por essa razão , Griffiths sugeriu o nome tricritico para esse ponto que anteriormen te era chamado de ponto crítico de segunda espécie.

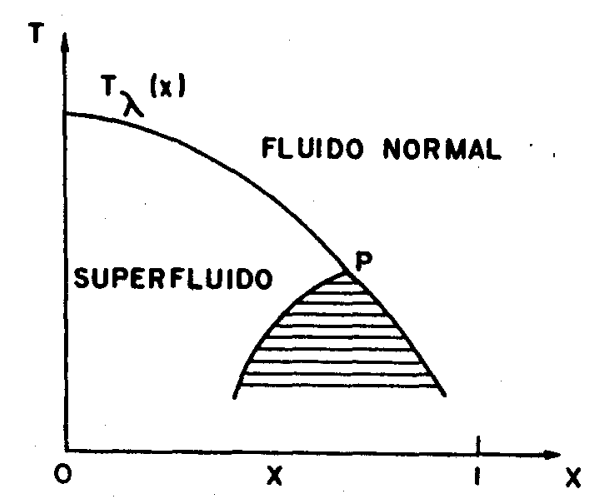

Figura III.1. Diagrama de fases da mistura $\mathrm{He}^{3}-\mathrm{He}^{4}$.

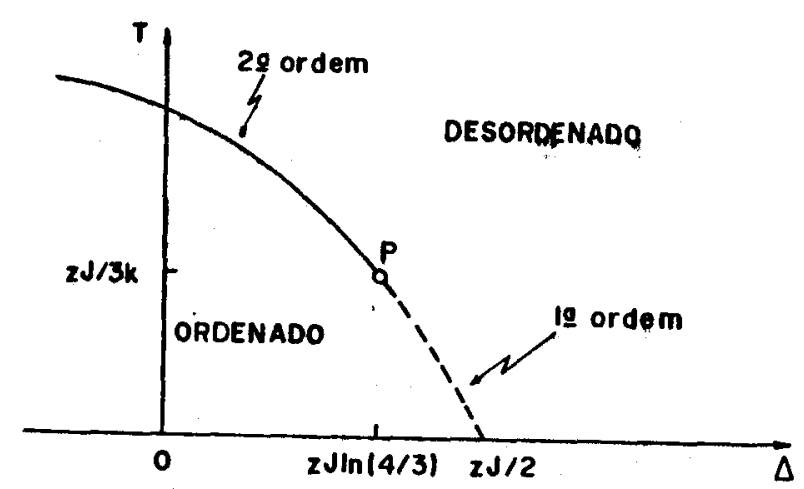

Figura III.2. Diagrama de fases do modelo de Blume-Capel de acordo com a teoria de campo médio.

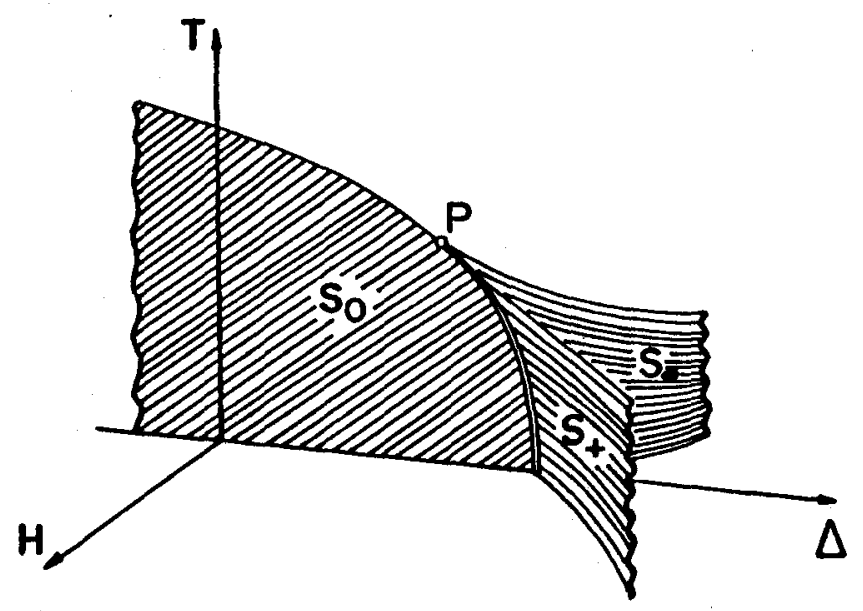

Figura III.3. Diagrama de fases do modelo de Blume-Capel depois da inclusão de um campo magnêtico. 
Neste trabalho nós não investigaremos o modelo de Blume Emery Griffiths (BEGl em duas dimensões mas sim, o seu anālogo quân tico unidimensional. Para isso, vamos supor que o modelo seja aniso trópico de forma que possamos fazer o limite de tempo continuo ${ }^{13}$. Assim, escrevendo o hamiltoniano na forma

$$
\begin{aligned}
H= & -J_{x} \sum_{i, j} S(i, j) S(i+1, j)-K_{x} \sum_{i, j} S^{2}(i, j) S^{2}(i+1, j) \quad(I I I .2) \\
& -J_{t} \sum_{i, j} S(i, j) S(i, j+1)-K_{t} \sum_{i, j} S^{2}(i, j) S^{2}(i+1, j)+\Delta \sum_{i, j} S^{2}(i, j)
\end{aligned}
$$

podemos colocar a matriz de transferência $T$ na forma

$$
T=e^{-\frac{H_{1}}{2}} e^{-H_{0}} e^{-\frac{H_{1}}{2}}
$$

onde

$$
\begin{aligned}
H_{0}= & x \sum_{i} S_{x}(i)+z \sum_{i} S_{x}^{2}(i) \\
H_{1}= & Y \sum_{i} S_{z}^{2}(i)-\beta J_{x} \sum_{i} S_{z}(i) S_{z}(i+1) \\
& -\beta K x \sum_{i} S_{z}^{2}(i) S_{z}^{2}(i+1)
\end{aligned}
$$

As quantidades $S_{x}(i)$ e $S_{z}(i)$ são agora operadores de spin 1 que atuam no espaço produto, isto ê

$$
S_{w}=\|\otimes\| \otimes \ldots \otimes \hat{S}_{N} \otimes \ldots \otimes \|, \quad N=x, z
$$




$$
\hat{S}_{x}=\frac{1}{\sqrt{2}}\left(\begin{array}{ccc}
0 & 1 & 0 \\
1 & 0 & 1 \\
0 & 1 & 0
\end{array}\right) \quad \hat{S}_{z}=\left(\begin{array}{ccc}
1 & 0 & 0 \\
0 & 0 & 0 \\
0 & 0 & -1
\end{array}\right)
$$

Os novos acoplamentos $\mathrm{X}, \mathrm{Y}$ e $\mathrm{z}^{25,26}$ que aparecem em $\mathrm{H}_{\mathrm{O}} \mathrm{e}$ $\mathrm{H}_{1}$ podem ser escritos em termos do acoplamentos originais como se gue:

$$
\operatorname{senh}^{2} x=e^{-\beta K_{t}}\left[\cosh \left(B J_{t}\right)-e^{\beta K_{t}}\right]^{-1},
$$

$$
e^{-2 z}=\frac{\cosh \left(\beta J_{t}\right)}{\operatorname{senh}^{2}\left(\beta J_{t}\right)}\left[\cosh \left(\beta J_{t}\right)-e^{-\beta K_{t}}\right],
$$

e

$$
y=\beta \Delta-\ln \left[2 e^{\beta K_{t}} \cosh \left(\beta J_{t}\right)\right]
$$

Podemos verificar facilmente que a matriz de transferên cia T (III.3) comuta com o operador paridade definido por:

$$
\begin{aligned}
& P=\prod_{i} \otimes\left[2 \hat{S}_{x}^{2}-\|\right] \\
& \rho^{2}=\|\otimes\| \otimes\|\otimes \ldots \otimes\|
\end{aligned}
$$

sendo exatamente essa invariância $\mathrm{z}(2)$ de $\mathrm{T}$ a simetria quebrada na transição.

Na base em que $S_{X}$ é diagonal a matriz de transferência $T$ terá uma forma bloco-diagonal. Os autovalores de $T$ são obtidos dia- 
gonalizando-se independentemente os dois blocos diagonais que săo caracterizados pela paridade (par ou impar) dos estados. Podemos, então, classificar os operadores do modelo em impares (que ligam es tados de paridade par com estados de paridade ímpar) e pares (que 'co nectam estados de mesma paridade). No primeiro conjunto estarão os operadores magnéticos (que se acoplam aos campos) e no segundo esta rão os térmicos.



Figura III-4a. Forma bloco-diagonal da matriz de transferência $T$, com setores caracterizados pela pa ridade.

Alēm da paridade, existem outras quantidades que são conservadas, o que possibilita colocar os dois setores de $\mathrm{T}$ (de parida de par e impar, conforme figura III-4a) numa forma bloco diagonal também (figuras III-4b e III-4c), onde esses novos blocos, de dimen são menor, são caracterizados pelos autovalores dessa quantidade conservada.

No caso de condições de contorno periódicas, o momentum $K=\frac{2 \pi}{L} h \quad(n=0,1, \ldots,[L / 2])$ é conservado, o que permite a divisão de cada um dos setores em $([L / 2]+1)$ subsetores $([\alpha])$ significa o maior número inteiro menor que $\alpha$ ) caracterizados pelos possíveis valores $\left(\frac{2 \pi}{l} n\right)$ do momentum. 


$$
\left(\begin{array}{c:c:c}
k_{1} & 0 & 0 \\
\hdashline 0 & k_{2} & 0 \\
\hdashline 0 & 0 & k_{3}
\end{array}\right)
$$

Figura III.4b. Forma bloco-diagonal de um setor de paridade defini da de $T$, com blocos caracterizados pelos valores $k_{1}, k_{2}$ e $k_{3}$ do mo mentum.

$$
\left(\begin{array}{c:c:c:c}
K_{1} & 0 & 0 & \\
\hdashline 0 & K_{2} & 0 & 0 \\
\hdashline 0 & 0 & K_{3} & \\
\hdashline & & & \text { PARIDADE } \\
& 0 & & \text { IMPAR }
\end{array}\right)
$$

Figura III-4c. Matriz de transferência $\mathrm{T}$ com estrutura bloco-diago nal, sendo os blocos caracteriza dos pela paridade e pelo momentum.

O mesmo acontece no caso de condições de contorno antiperiōdicas, só que agora o momentum é $k=\frac{\pi}{L}(2 n+1) \quad(n=0,1, \ldots,[(L-1) / 2])$ no setor impar.

Com condições livres de contorno pode-se explorar a simetria por reflexão, que permite uma divisão em dois subsetores carac terizados pela reflexão positiva ou negativa dos estados.

Essa divisão em setores, da matriz de transferência T', traz muitas vantagens do ponto de vista computacional, uma vez que cada um dos blocos pode ser diagonalizado separadamente. Dessa forma é possivel ter acesso a um maior número de autovalores, com mais precisão e menor tempo de CPU.

Em princípio $T$ já é um operador quântico unidimensional que, uma vez diagonalizado, fornecerã a função de partição e o comprimento de correlação. Entretanto, devemos lembrar que a matriz de transferência é uma exponencial de operadores e tem, portanto, vā rias potências do expoente. Isso significa que quase todos os ele mentos da matriz $\mathrm{T}$ serão diferentes de zero o que, somado ao räpido 
crescimento da sua dimensão com o tamanho do sistema ( $\left.3^{L}\right)$, torna praticamente inviāvel a sua diagonalização. Por essa razão, traba lharemos com a versão quântica do modelo. Isso corresponde a tomarmos o limite altamente anisotrópico do modelo no qual $X, Y, Z, J_{X}$ e $K_{X}$ são todos infinitesimais. Para se conseguir isso é preciso escolher

$$
\beta J_{x}, \beta K_{x}<1
$$

e simultâneamente

$$
\beta J_{t}, \beta K_{t} \gg 1
$$

sob a restrição

$$
\beta\left(\Delta-J_{t}-K_{t}\right)<1
$$

Satisfeitas essas condições a matriz de transferēncia pode ser escrita como

$$
T \simeq \exp \left\{-\beta J_{x} H\right\},
$$

com

$$
\begin{aligned}
H= & -\sum_{i}\left\{S_{z}(i) S_{z}(i+1)+\alpha S_{z}^{2}(i) S_{z}^{2}(i+1)\right. \\
& \left.-\delta S_{z}^{2}(i)-\gamma S_{x}(i)-\lambda S_{x}^{2}(i)\right\}
\end{aligned}
$$

e

$$
\alpha=\frac{K x}{J_{x}} \quad, \quad \delta=\frac{y}{\beta J_{x}} \quad, \quad y=\frac{x}{\beta J_{x}}, \quad \lambda=\frac{Z}{\beta J_{x}}
$$


E claro que, como H tem a mesma dimensão que $T$, podería mos pensar que a dificuldade com a diagonalização continua a mesma. Entretanto, 'se analisarmos em detalhe o operador 11 , veremos que este só liga estados com no māximo 2 spins diferentes e isso faz com que cada linha de $3^{L}$ elementos possua apenas $L$ elementos não nulos. Essa matriz "rarefeita" pode então ser diagonalizada pelo método de Lanczos $^{27}$ (ver apêndice B) que é um método muito eficiente para se calcular os autovalores extremos de uma matriz.

2. O DIAGRAMA DE FASES

Na formulação hamiltoniana a hipōtese do FSS assegura que a diferença de energia entre o estado fundamental e o primeiro esta do excitado (correspondente ao inverso do comprimento de correla ção) de um sistema infinito

$$
G(\beta)=E_{1}-E_{0}
$$

que vai a zero no acoplamento critico segundo

$$
G(\beta) \sim\left(\beta-\beta_{c}\right)^{\gamma}
$$

deve se comportar como

$$
G_{L}\left(\beta_{C}\right) \sim L^{-1}
$$

no caso de um sistema de tamanho L.

A equação (III.18) sugere que o acoplamento crîtico pode ser encontrado extrapolando a sequência de valores $\beta^{*}$ que satisfazem a igualdade 


$$
L G_{L}\left(\beta^{*}\right)=L^{\prime} G_{L}\left(\beta^{*}\right)
$$

Em 1985, Alcaraz et al ${ }^{28}$ obtiveram o diagrama de fases do modelo BEG utilizando a equação (III.19). A localização do ponto tricrítico foi feita atravēs de uma equação que generaliza (III.19), isto é:

$$
L \tilde{G}_{L}\left(\beta_{t}\right)=L^{\prime} \tilde{G}_{L}^{\prime}\left(\beta_{t}\right)
$$

onde $\tilde{G}_{L}$ é a diferença de energia entre o estado fundamental e o se gundo estado excitado (segundo "gap"l .

Justificamos o uso dessa equação lembrando que um ponto tricrítico è o ponto onde três fases (que antes coexistiam) tornamse idênticas e, portanto, devemos ter degenerescência dos três primeiros autovalores. A degenerescência, porém, só ocorre no limite de $L=\infty$ e, assim, a equação (III.20) fornece uma estimativa do acoplamento tricrítico.

Neste trabalho nôs utilizaremos o diagrama de fases obtido por Alcaraz et $a 1^{28}$, para investigar o comportamento crítico do modelo de Blume-Capel através do seu espectro.

\section{CALCULO DO NUMERO DE ANOMALIA CONFORME C}

Para determinar o número de anomalia conforme $\mathrm{C}$ para $\circ$ mo delo de Blume-Cape1, lembramos que as correçōes devido ao tamanho finito do sistema ao autovalor mais baixo $E_{o}$ do hamiltoniano estão relacionadas à "C" (ver seção 3, Cap. IIl através da relação ${ }^{12}$ 
No caso de condições de contorno periōdicas, f é o limite termodinàmico da energia do estado fundamental por sitio e $\psi$ é uma constante $\left(f=\left(\beta J_{x}\right)^{-1}\right.$, conforme (III.14)) que serve para restau rar a invariância conforme perdida no limite anisotröpico. Para encontrar o valor de $\mathrm{f}$ é preciso obter a energia do estado fundamen tal por sítio de cadeias finitas e extrapolar para o limite termodi nâmico $(L \rightarrow \infty)$. O fator é obtido calculando-se o "gap" que separa as energias mais baixas do setor impar, com momentos respectivamente iguais a zero e $2 \pi / L$ (este "gap" é igual à $2 \pi / / L$ ).

Nós calculamos o valor de $\mathrm{C}$ no ponto tricrítico $\mathrm{T}$ e num ponto s sobre a linha critica de 2 a.ordem (veja figura III.1). Na tabela I estão listadas as estimativas da energia do estado funda mental por sitio $\left(E_{0} / L\right)$, e nas tabelas II e III, respectivamente , estão as estimativas do fator $\gamma$ e do número de anomalia conforme $C$ em ambos os casos. O s valores extrapolados da tabela III concordam com os valores esperados $\mathrm{C}=7 / 10(\mathrm{~m}=4)$ no ponto tricritico e $\mathrm{C}=1 / 2$ $(m=3)$ no ponto $s$ onde a transição é do tipo Ising.

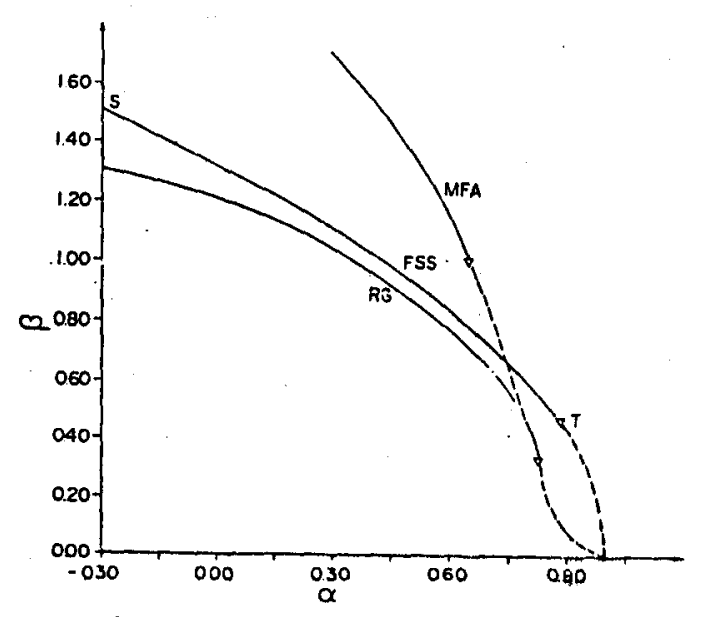

Figura III.1. Diagrama de fases para o hamiltoniano de Blume-Capel obtido por Alcaraz et al ${ }^{28}$ usando a técnica do finite-size scaling. O ponto tricrítico $\mathrm{T}(=0.9103$, $=0.4155)$ separa as linhas de transição de la.ordem (li-

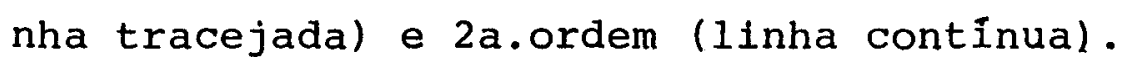


o caso $C=1 / 2$ já foi suficientemente estudado uma vez que o modelo de Ising é exatamente solüvel para qualquer valor de L. 0 espectro pode ser obtido sob vārias condições de contorno e todos os gaps podem ser explicados em termos das dimensões $1 / 16,1 / 2$ e 0 associadas ao inteiro $\mathrm{m}=3$. A situação no caso $\mathrm{C}=7 / 10$ é porém bastan te diferente, uma vez que não hã soluções exatas disponíveis e tudo o que se conhece è o conjunto dos expoentes criticos conjecturados por Nienhuis 20 . No próximo capítulo analisaremos o espectro da cadeia de Blume-Capel sujeita a uma variedade de condições de contorno para elucidar algumas questões ainda pendentes a respeito do seu comportamento critico.

\begin{tabular}{lll}
\hline$L$ & $\left(-\frac{E_{O}}{L}\right)$ no ponto $T$ & $\left(-\frac{E_{O}}{\mathrm{~L}}\right)$ no ponto $S$ \\
\hline 2 & 0.23377843 & 1.90861248 \\
3 & 0.20175177 & 1.85737302 \\
4 & 0.19114537 & 1.84078977 \\
5 & 0.18633838 & 1.83335518 \\
6 & 0.18375409 & 1.82938124 \\
7 & 0.18220498 & 1.82700715 \\
8 & 0.18120324 & 1.82547517 \\
9 & 0.18051812 & 1.82442889 \\
\hline ext. & $0.1779512(2)$ & $1.820520(5)$ \\
\hline
\end{tabular}

TABELA I : Energia do es tado fundamental por sitio do hamiltoniano de Blume-Capel no ponto $T$ e no ponto $s$. 


\begin{tabular}{ccc}
\hline $\mathrm{L}$ & $\mathrm{T}$ & $\mathrm{S}$ \\
\hline 2 & 0.373025 & 0.689156 \\
3 & 0.478083 & 0.932940 \\
4 & 0.517130 & 1.038314 \\
5 & 0.535363 & 1.092954 \\
6 & 0.545205 & 1.124687 \\
7 & 0.551066 & 1.144629 \\
8 & 0.554811 & 1.157925 \\
9 & 0.557290 & 1.167207 \\
\hline
\end{tabular}

TABELA II: Sequência de esti mativas para o fator $y$ nos pontos $\mathrm{T}$ e $\mathrm{S}$.

\begin{tabular}{lll}
\hline $\mathrm{L}$ & $\mathrm{C}$ - ponto $\mathrm{T}$ & $\mathrm{C}$-ponto $\mathrm{S}$ \\
\hline 2 & 1.143451 & 0.976517 \\
3 & 0.855933 & 0.678981 \\
4 & 0.780001 & 0.596528 \\
5 & 0.748558 & 0.560692 \\
6 & 0.732563 & 0.541680 \\
7 & 0.723441 & 0.530339 \\
8 & 0.717773 & 0.523017 \\
9 & 0.712548 & 0.518008 \\
\hline ext. & $0.70(2)$ & $0.49(7)$ \\
\hline
\end{tabular}

TABELA III: Sequência de es timativas para o número de anomalia conforme (C) da ca deia quântica de BlumeCapel. 


\section{CAPITULO IV}

EFEITO DAS CONDIÇÕES DE CONTORNO SOBRE O ESPECTRO DO MODELO DE BLUME-CAPEL

Na Seção 5 do Capítulo II verificamos as predições da in variância conforme no modelo de Ising, cujos autovalores são conhecidos exatamente. Neste Capítulo nós obtemos o espectro do modelo de Blume-Capel, no ponto tricritico (T), usando diferentes tipos de condições de contorno.

- Nesse ponto o espectro inteiro deve ser descrito pelas representações irredutíveis da álgebra de virasoro associada com $\mathrm{C}=$ = 7/10 cujas dimensões, fornecidas pela fórmula de Kac, são:

$$
\begin{array}{lll}
0 & \\
7 / 16 & 3 / 80 \\
1 / 10 & 3 / 5 & 3 / 2
\end{array}
$$

A parte "experimental" do trabalho consiste no cálculo dos "gaps" escalados:

$$
\xi_{i}=\lim _{L \rightarrow \infty} \frac{L}{2 \pi l}\left(E_{i}^{L}-E_{0}^{L}\right)
$$

sendo $E_{0}^{L}$, a energia do estado fundamental, $E_{i}^{L}$ o i-ésimo estada excitado e $f$ a constante multiplicativa a ser extraída do espectro. o hamiltoniano H utilizado para se obter os autovalores foi escrito na forma

$$
\dot{H}=-\sum_{i=1}^{L}\left\{S_{z}(i) S_{z}(i+1)-\alpha S_{z}^{2}(i)-\beta S_{x}(i)\right\}
$$


Lembramos do Capitulo III que este hamiltoniano comuta com o operador paridade

$$
\rho=\prod_{i} \otimes\left[2 S_{x}^{2}(i)-11\right]
$$

- que permite a divisão do espectro em dois setores (par e impar) . os "gaps" escalados serão denotados por $\xi_{i}^{(+)}$ou $\xi_{i}^{(-)}$conforme a pa ridade do estado excitado $E_{i}$.

Um estudo do efeito das condições de contorno sobre o es pectro pode conduzir ao perfeito entendimento dos operadores que participam da teoria bem como da estrutura e relacionamento entre eles.

Gehlen e Rittenberg ${ }^{(21)}$ e Cardy ${ }^{(22)}$ analisaram o espec tro do modelo de Potts-3 estados usando diferentes tipos de condi ções de contorno. Para cada uma delas eles identificaram um subgrupo diferente das dimensões de escala fornecidas pela fórmula de Kac Além disso, valores de $p$ e q que com um tipo de condição de contorno correspondiam a operadores do tipo energia em outro caso estavam associadós a operadores do tipo magnetização e vice-versa.

Nas seções seguințes, mostraremos os resultados obtidos pela análise do hamiltọniano $\mathrm{H}$ sujeito a quatro diferentes condi ções de contorno: periōdicas, livres, fixas e antiperiódicas.

1. CONDIÇÕES PERIODICAS DE CONTORNO

No caso de condições periôdicas de contorno o grupo de simetria ê $G=T \otimes \bar{T}$ onde $\bar{T}(\bar{T})$ é o grupo das substituições analíticas da variāvel $z(\bar{Z})$ e por essa razão as dimensões de escala $X$ são somas de dois h's $(X=h+\bar{h})$. Devido à invariância translacional apenas operadores com spin inteiro podem ocorrer, o que significa , 
nesse caso, que $h=\bar{h}$. Assim, o número de representações irredutiveis descrevendo o espectro é seis: $(0,0),(3 / 80,3 / 80),(7 / 16,7 / 16)$, $(1 / 10,1 / 10),(3 / 5,3 / 5)$ e $(3 / 2,3 / 2)$.

As condições periódicas de contorno exigem que

$$
S_{z}(L+1)=S_{z}(1)
$$

0 espectro foi dividido em setores caracterizados pela paridade e pelo momento K. Para selecionar os estados com um determinado valor do momento é preciso construir um vetor de onda ini cial com esse valor de $K$. Devido à simetria $z(2)$ do modelo, os valo res de $K$ permitidos serão múltiplos de $(2 \pi / L)$, analogamente ao mode lo de Ising (seção 5, Cap.II), submetidoṣ às mesmas condições de contorno. O vetor de onda inicial é da forma

$$
\frac{1}{N_{O R M}}\left(a_{1} \phi_{1}+a_{2} \phi_{2}+\cdots+a_{2} \phi_{2}\right)
$$

onde $\phi_{i}$ é a translação cỉclica $(j=L+1=1)$ por $(i-1)$ espaçamentos de rede, $a_{i}$ é o coeficiente que carrega o momento $k$,

$$
a_{i}=\cos \left(\frac{2 \pi m}{L}(i-1)\right)
$$

e NORM é o fator de normalização

$$
\text { NORM }=\left[\sum_{i=1}^{L} a_{i}^{2}\right]^{1 / 2}
$$

Usando o mêtodo de Lanczos (apêndice B) para a diagonalí zação de $\mathrm{H}$ conseguimos analisar redes de até 9 sítios. Os "gaps" es calados $\xi_{i}$ foram, então, obtidos usando-se o método de extrapolação de Van der Broeck-Schwardz. A constante pode ser obtida do "gap" 
entre as energias mais baixas do setor impar, com momento $K=0$ e $K=$ $=2 \pi / L, j a ̄$ que, de acordo com as predições da invariância conforme, o momento $\mathrm{K}$ e as energias $\mathrm{E}_{\mathfrak{i}}$ do espectro são dadas por

$$
K=(m-\bar{m}) \frac{2 \pi}{L}, \quad E_{i}=\frac{2 \pi}{L}\left(x_{i}+m+\bar{m}\right) \gamma
$$

Assim, os estados de energia mais baixa para uma dada di mensão $\chi_{i}$ com momentos $K=0$ e $K=2 \pi / L$, correspondem, respectivamente, aos seguintes valores de $m$ e $\bar{m}: m=\bar{m}=0$ e $m=1, \bar{m}=0$, de forma que:

$$
E_{i}\left(K=\frac{2 \pi}{L}\right)-E_{i}(K=0)=\frac{2 \pi}{L} \gamma
$$

Os resultados "experimentais" do espectro com condições periódicas de contorno estão na Tabela $I$, juntamente com as repre sentações do grupo de simetria $G=\uparrow \otimes \bar{\Gamma}$ * .

* Como se pode ver na tabela todos os "gaps" podem ser explicados por

$$
\xi_{i}(K)=(h+m)+(\bar{h}+\bar{m})
$$

com

$$
K=(h+m)-(\bar{h}+\bar{m})
$$

onde $\mathrm{m} e \overline{\mathrm{m}} \epsilon_{\mathrm{N}}$. 
TABELA I - Espectro experimental $\xi_{i}(K)$ com sua interpre tação teōrica.

\begin{tabular}{|c|c|c|c|c|c|c|c|c|c|}
\hline K & $h+m+\bar{h}+\bar{m}$ & $(0,0)$ & $(3 / 80,3 / 80$ & $(7 / 16,7 / 16)$ & $(1 / 10,1 / 10)$ & $(3 / 5,3 / 5)$ & $(3 / 2,3 / 2)$ & $\xi_{i}^{(+)}(\mathrm{K})$ & $\xi_{i}^{(-)}(K)$ \\
\hline \multirow[t]{8}{*}{0} & 0.075 & - & 1 & - & - & - & - & & $0.075(1)$ \\
\hline & 0.2 & - & - & - & 1 & - & - & 0.19911 & \\
\hline & 0.875 & - & - & 1 & - & - & - & & $0.87(6)$ \\
\hline & 1.2 & - & - & - & - & 1 & - & $1.18(7)$ & \\
\hline & 2.075 & - & 1 & - & - & - & - & & $2.07(7)$ \\
\hline & 2.2 & - & - & - & 1 & - & - & $2.19(2)$ & \\
\hline & 2.875 & - & - & 1 & - & - & - & & $2.8(5)$ \\
\hline & 3.0 & - & - & - & - & - & 1 & $3.0(6)$ & \\
\hline \multirow[t]{3}{*}{1} & 1.075 & - & 1 & - & - & - & - & & $1.075(6)$ \\
\hline & 1.2 & - & - & - & 1 & - & - & $1.1(9)$ & \\
\hline & 1.875 & - & - & $i$ & - & - & - & & $1.87(8)$ \\
\hline \multirow[t]{2}{*}{2} & 2.0 & 1 & - & - & - & - & - & 2.0 & \\
\hline & 2.075 & - & 1 & - & - & - & - & & $2.0(7)$ \\
\hline
\end{tabular}

2. CONDIÇÕES ANTIPERIÓDICAS DE CONTORNO (CAC)

As condições de contorno utilizadas se refletem no hamiltoniano através do termo $s_{L}^{Z} s_{L+1}^{Z}$ ou mais precisamente em $s_{L+1}^{Z}$. No ca so de condições antiperiôdicas de contorno $s_{L+1}^{Z} \bar{e}$ associado à $s_{1}^{Z}$ por

$$
S_{L+1}^{z}=\rho^{-1} S_{1}^{z} \rho
$$

onde $P_{e}$ o operador paridade 


$$
P=\prod_{i=1}^{L}\left(2 s_{x}^{2}-\|\right),
$$

que implementa a simetria $Z(2)$.

E fácłl verificar que (IV.11) implica em

$$
S_{L+1}^{z}=-S_{1}^{z}
$$

que no hamiltoniano significa trocar $J$ por $-J$ no contorno.

Da mesma forma que no caso de condições periódicas de contorno, o espectro è dividido em setores caracterizados pela paridade e pelo momento $\mathrm{K}$. A û́nica diferença è que no setor Impar os valores permitidos de $K$ serão múltiplos impares de $\pi / L$, isto $\bar{e}, K=(2 m+1) \pi / L$ * os "gaps" escalados $\xi_{1}$ para esse caso estão na Tabela II. Vejamos como esses "gaps" estão relacionados com os operadores do mo delo. Devido à simetria $Z(2)$ existente, os resultados obtidos podem ser explicados de forma anâloga ao modelo de Ising.

\begin{tabular}{llll}
\hline$x+m$ & $\xi_{i}^{+}$ & $\xi_{i}$ \\
\hline $4 / 5$ & 0 & $0.79(2)$ & \\
$5 / 8$ & 0 & & $0.62(6)$ \\
0 & 1 & $0.99(8)$ & \\
$57 / 40$ & 0 & & $1.42(8)$ \\
$5 / 8$ & 1 & & $1.6(3)$ \\
$4 / 5$ & 1 & $1 .(7)$ & \\
\hline
\end{tabular}

TABELA II - Espectro experimental $\xi_{i}$ para o caso de condições antiperiődicas de contorno.

* Isto acontece porque neste setor só é possivel excitar um número impar de "partỉculas" (veja mais detalhes no que foi apresentado sobre o modelo de Ising, seção 5, Cap.II). 
Com esse tipo de condição de contorno conseguimos identí ficar a dimensão de escala $\chi \psi$ do operador fermiônico $\psi$ (que possui $\operatorname{spin}=\mathrm{h}-\overline{\mathrm{h}}=1 / 2$ ) refletindo a supersimetria do modelo (simetria en tre bósons e fêrmions). Lembramos que para o modelo de Ising as con dições antiperiódicas de contorno podem ser obtidas pela introdução de operadores desordem de forma que o "gap" dentro das condições an tiperiódicas de contorno è obtido subtraindo-se da dimensão do operador em questão a dimensão do operador desordem. Lembramos ainda que no caso do Ising o estado fundamental (com condições periódicas de contorno) do setor impar, é igual ao estado fundamental do setor par no caso de condições antiperiódicas (CAC) que, portanto, o operador desordem tem a mesma dimensão de escala do operador magnetiza ção - Extrapolando esses resultados para o modelo de Blume-Capel, a dimensão do operador desordem, è $x_{\mu}=3 / 40$. Se somarmos, então, essa dimensão $X_{M}$ da dimensão de escala associada ao 18 "gap" nas condições antiperiódicas de contorno teremos a dimensão do operador fermiônico $\psi$. Da Tabela II vemos que isso será

$$
x_{\psi}=x_{1}+x_{\mu} \simeq 0.63+3 / 40 \simeq 0.705 \simeq 7 / 10
$$

Das dimensões de escala fornecidas pela fórmula de Kac (II.25) para esse modelo, vemos que essa dimensão $\chi \psi$ corresponde às representa ções $(3,2)$ e $(3,3)$, isto é

$$
x_{\psi}=h_{3,3}+h_{3,2}=1 / 10+3 / 5=7 / 10
$$

Podemos certificar o resultado calculando o spin, que pa ra o operador fermiônico deverâ ser $s=+1 / 2$. E o resultado é

$$
s=h_{3,2}-h_{3,3}=3 / 5-1 / 10=1 / 2,
$$


como esperāvamos.

Recentemente, Cardy obteve soluções para as regras de so ma de inversão, no caso de CAC, formalmente escritas como:

$$
[M \otimes M] N_{A}(p, q ; \bar{p}, \bar{q})=d_{A}(p, q ; \bar{p}, \bar{q})
$$

o resultado é que os operadores contidos nesse espectro devem obedecer à restrição:

$$
N_{A}(p, q ; \bar{p}, \bar{q})=\delta p, m-\bar{p} \delta q \bar{q},
$$

o que leva às seguintes dimensões de escala possiveis:

$$
\begin{array}{ll}
\mathcal{W}(2,1 ; 2,1)=1 & x=7 / 8 \\
N_{A}(2,2 ; 2,2)=1 & x=3 / 40 \\
N_{A}(3,1 ; 1,1)=1 & x=3 / 2 \\
\mathcal{N}_{A}(3,2 ; 3,3)=1 & x=7 / 10
\end{array}
$$

Além disso, ele obteve que valores de $\mathrm{p}$ pares (impares) estão associados a operadores pares (Impares) de simetria Z(2). Assim, subtraindo a dimensão de escala do operador desordem $\chi_{\mu}=3 / 40$ das dimensões dadas em (IV.19) obtēm-se que as dimensões dentro das condições antiperiōdicas são:

$$
x=7 / 8-3 / 40=8 / 10
$$

e

$$
x=3 / 40-3 / 40=0
$$


no setor par, e

$$
x=7 / 10-3 / 40=5 / 8=0.625
$$

e

$$
x=3 / 2-3 / 40=57 / 40=1.425 \text {, }
$$

no setor Impar.

Estes resultados estão esquematizados na Figura 1.

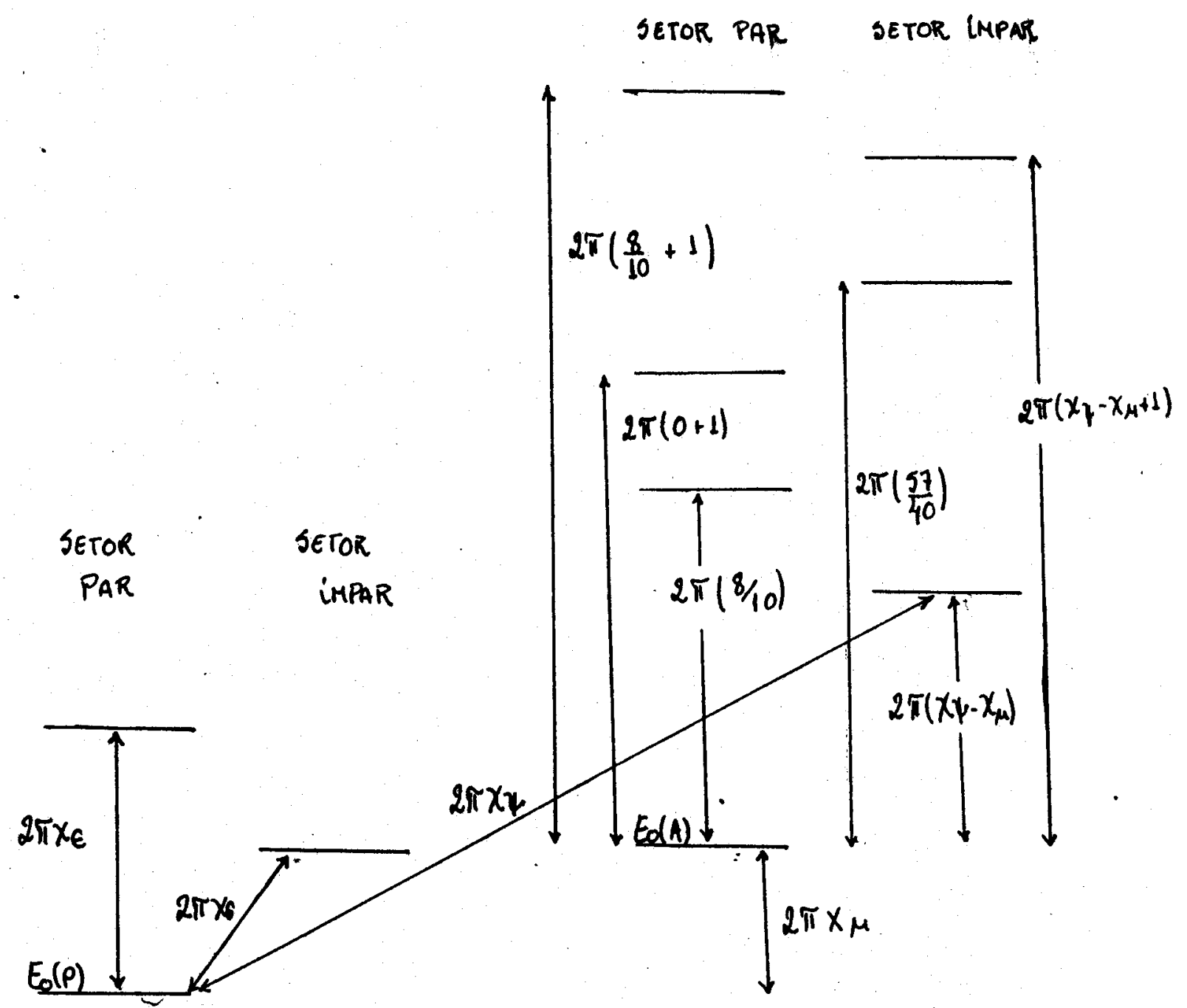

CONDIÇÕES PERIÓdicAs

CONDIÇÕES ANTIPERIÓdicAs

Figura 1 - Ilustração das excitações mais baixas do espec tro. $x_{\gamma}$ e $x_{\mu}$ denotam as dimensões de escala dos operadores fermiônico e desordem, respecti vamente. 
3. CONDIÇÃO LIVRE DE CONTORNO

Neste caso, os autovalores de $\hat{\mathrm{H}}_{\mathrm{F}}$ determinam expoentes criticos da superficie ${ }^{29}$ (Binder, 1983) jâ que a invariância conforme dá informaçōes sobre as funções de correlação ao longo da fronteira do sistema ${ }^{30}$. Impor condições Iivres de contorno, significa des vincular o parâmetro de ordem nas extremidades do sistema, de forma que a interação $\mathrm{s}_{\mathrm{L}}^{\mathrm{Z}} \mathrm{s}_{\mathrm{L}+1}^{\mathrm{Z}}$ deixa de existir no Hamiltoniano.

A transformação que relaciona essa geometria àquela de um sistema semi-infinito, relactona também os autovalores de $\hat{\mathrm{H}}_{\mathrm{F}}$ com os expoentes criticos que descrevem o decaimento das correlações no contorno. A equação é

$$
E_{m}(F)-E_{0}(F)=\frac{\pi}{L}\left(X_{s}+m\right), \quad m=0,1, \ldots
$$

onde o expoente de superfície $X_{S}$, nesse caso, coincide com a dimen são h da representação da álgebra de virasoro correspondente. E importante mencionar que no caso de condições livres de contorno exis te apenas uma àlgebra de virasoro e, assim, o espectro pode ser obtido atravésde inteiros $N_{F}(p, q)$ (caracterizados por sô un par de nú meros $p, q)$.

As estimativas da dimensão anômala $\chi_{s}$ do operador ordem na superfície estão na Tabela III. O resultado para $\eta_{11}=2 x_{3}=3$, è com patível com a equação $\eta_{\|}=2 /(3 \gamma-1)$ deduzida por cardy ${ }^{30}$ para o modelo de Potts-escalar. A coincidência não é surpreendente uma vez que os dois modelos estão relacionados por uma continuação analítica em torno de $q=4^{31}$. 
soma para a inversão não são suficientes para determinar completa mente o espectro de $\hat{\mathrm{H}}_{\mathrm{F}}$. Em função disso ele encontrou $\mathcal{W}_{\mathrm{F}}(1,1)=$ $=d f_{F}(3,1)=1$ e $\int_{F}(1,2)=\int_{F}(3,2)=I$, com $I=0$ ou 1. A possibilidade $I=1$ foi eliminada considerando que dela resultaria um operador expúreo (par) relevante, associado à $(3,2) \operatorname{com} \tilde{\chi}=3 / 5$. Escolhendo então $I=0$, obtém-sed $\int_{F}=1$ apenas para as representações $(1,1)$ e $(3,1)$ que correspondem à $h=0$ e $3 / 2$.

\section{CONDIÇÃO FIXA DE CONTORNO}

Como a variävel de $\operatorname{spln} S_{1}$ pode assumir três valores diferentes $\left(s_{1}=+1,0,-1\right)$, que nōs representaremos por,$+-e 0$, podemos formar vārios setores fixando uma extremidade da rede no estado $\mathrm{X}$ e a outra no estado $Y \cdot(X, Y=+,-$ ou 0$)$. Mas devido à simetria $Z(2)$ ape nas três setores distintos aparecem: $\mathrm{H}_{++}, \mathrm{H}_{+-}$e $\mathrm{H}_{\mathrm{O}_{+}} \cdot$

Os setores $\mathrm{H}_{++}$e $\mathrm{H}_{+-}$foram identificados com os setores par e impar, respectivamente, das condições livres de contorno (ver Tabela V), Novamente, isso reflete a simetria $\mathrm{Z}(2)$ do modelo, já que no modelo de Ising, os casos de condições fixas e livres de con torno são relacionadas exatamente por uma transformação de dualidade. Vejamos isso melhor: a matriz de transferência para condições livres do modelo de Ising é

$$
\hat{T}_{F}=\exp \left(J \sum_{j=1}^{L-1} \sigma_{j}^{z} \sigma_{j+1}^{z}\right) \exp \left(J \sum_{j=1}^{L} \sigma_{j}^{x}\right)
$$

uma transformação de dualidade è implementada definindo variāveis de desordem dadas por

$$
\begin{aligned}
& \mu_{j}^{x}=\sigma_{j}^{z} \sigma_{j+1}^{z} \quad(1 \leqslant j \leqslant L-1) \\
& \mu_{j}^{z}=\sigma_{1}^{x} \sigma_{2}^{x} \ldots \sigma_{j}^{x}
\end{aligned}
$$


TABELA III - Estimativas da dimensão de escala do opera dor magnetizaçäo na superfície.

\begin{tabular}{cc}
$\mathrm{L}$ & $\mathrm{X}_{3}$ \\
\hline 2 & 1.664915 \\
3 & 1.622975 \\
4 & 1.596981 \\
5 & 1.579588 \\
6 & 1.567248 \\
7 & 1.558093 \\
8 & 1.551061 \\
9 & 1.545508 \\
extr. & $1.50(2)$
\end{tabular}

Outras excitações do espectro foram obtidas considerando se os outros "gaps". Os resultados estão listados na Tabela IV.

TABELA IV - Espectro experimental no caso de condição li vre de contorno. Os números narcancio a con tribuição de cada h indicam a degenerescên cia encontrada "experimentalmente".

\begin{tabular}{lllll}
\hline $\mathrm{h}+\mathrm{m}$ & 0 & $3 / 2$ & $\xi_{i}^{(+)}$ & $\xi_{1}^{(-)}$ \\
\hline 1.5 & - & 1 & & $1.50(2)$ \\
2.5 & - & 1 & & $2.55(5)$ \\
3.0 & 1 & - & $3.00(1)$ & \\
3.5 & - & 2 & & $3.52(7), 3.54(7)$ \\
4.0 & 2 & - & $\begin{array}{l}3.9(8) \\
3.99(3)\end{array}$ \\
\hline
\end{tabular}

Vemos que o espectro é descrito, nesse caso, somente por duas representações ( 0 e 3/2), confirmando os resultados obtidos re centemente por cardy que investigou as soluções de $M \rho_{F}(p, q)=$ $=d P_{F}(p, q)$ para essa teoria. Nesse caso as condições das regras de 
em termos das quais a matriz $\hat{\mathrm{T}}_{\mathrm{F}}$ pode ser escrita*:

$$
\hat{T}_{F}=\exp \left(\mu_{1}^{z}+\mu_{i}^{z} \mu_{2}^{z}+\cdots+\mu_{L-1}^{z} \sum\right) \exp \left(\sum_{j=1}^{L-1} \mu_{j}^{x}\right)
$$

onde $\sum \equiv \prod_{j} G_{j}^{x}$ é o operador magnetização que implementa a simetria $z(2)$. Dessa relação pode-se concluir que o setor $\Sigma=+1$ de $\hat{H}_{F}$ è e quivalente $\overline{\mathrm{a}} \hat{\mathrm{H}}_{++}$e o setor $\sum=-1 \mathrm{a} \hat{\mathrm{H}}_{+-}$, isto é, o espectro é o mesmo nos dois casos.

TABELA V - Espectro "experimental" no caso de condiçăo fixa de contorno. O número marcando a contribui ção da dimensão $h$ indica a degenerescência obti da "experimentalmente".

\begin{tabular}{ccccc}
\hline $\mathrm{h}+\mathrm{m}$ & 0 & $3 / 2$ & $\xi_{i}^{++}$ & $\xi_{i}^{+-}$ \\
\hline 1,5 & - & 1 & & $1,50(0)$ \\
2,0 & 1 & - & 2,0 & \\
2,5 & - & 1 & & $2,5(2)$ \\
3,0 & 1 & - & $2,9(8)$ & \\
3,5 & - & 2 & & $3 .(5), 3.4(9)$ \\
4,0 & 2 & - & $3,9(8), 3 .(9)$ \\
4,5 & - & 2 & & $4,(4), 4 .(5)$ \\
\hline
\end{tabular}

* Na verdade os fatores estão na ordem invertida, mas o resultado é equivalente por uma transformação de similaridade. 


\section{COMENTARIOS FINAIS}

o estudo numérico do Hamiltoniano quântico asso ciado ao modelo de Blume-Capel permitiu identificar todos os $\underline{o}$ peradores da teoria com suas respectivas dimensões, além de confirmar alguns resultados obtidos por cardy ${ }^{22}$ com base na in variância modular. A semelhança com o modelo de Ising (que tam bém tem simetria $z(2)$ ) possibilitou o entendimento completo dos efeitos das värias condições de contorno sobre o espectro, que estão reunidos na tabela a seguir.

Cumpre ressaltar que um estudo preliminar das cor reções ao "scaling" não revelou informações relevantes. Esse porém, è um ponto que pretendemos retomar brevemente. A ques tão da supersimetria, que foi relegada a um segundo plano nessa dissertação, também é de nosso interesse. 


\begin{tabular}{|c|c|c|c|c|}
\hline $\begin{array}{l}\text { condição de } \\
\text { contorno }\end{array}$ & $\begin{array}{l}\text { nümerns quānticos } \\
(p, q ; \bar{p}, \bar{q})\end{array}$ & $\begin{array}{l}\text { Dimensões } \\
(\mathrm{h}, \overline{\mathrm{h}})\end{array}$ & Gap & Simetria \\
\hline \multirow{6}{*}{ periódica } & $(1,1 ; 1,1)$ & $(0,0)$ & & + \\
\hline & $(2,2 ; 2,2)$ & $(3 / 80,3 / 80)$ & $3 / 20$ & - \\
\hline & $(3,3 ; 3,3)$ & $(1 / 10,1 / 10)$ & $2 / 5$ & + \\
\hline & $(2,1 ; 2,1)$ & $(7 / 16,7 / 16)$ & $7 / 4$ & - \\
\hline & $(3,2 ; 3,2)$ & $(3 / 5,3 / 5)$ & $12 / 5$ & + \\
\hline & $(3,1 ; 3,1)$ & $(3 / 2,3 / 2)$ & 6 & + \\
\hline \multirow{4}{*}{ antiperiódica } & $(2,1 ; 2,1)$ & $(7 / 16,7 / 16)$ & $8 / 5$ & + \\
\hline & $(3,3 ; 3,2)$ & $(1 / 10,3 / 5)$ & $7 / 5$ & - \\
\hline & $(1,1 ; 1,1)$ & $(0,0)$ & & + \\
\hline & $(3,1 ; 1,1)$ & $(3 / 2,0)$ & $57 / 20$ & - \\
\hline \multirow[t]{2}{*}{ livre } & $(1,1)$ & 0 & 2 & + \\
\hline & $(3,1)$ & $3 / 2$ & $3 / 2$ & - \\
\hline \multicolumn{5}{|l|}{ fixa } \\
\hline t+ & $(1,1)$ & 0 & 2 & \\
\hline+- & $(3,1)$ & $3 / 2$ & $3 / 2$ & \\
\hline
\end{tabular}

TABELA VI - A 2a.coluna mostra os nūmeros quânticos (pq; $\overline{p q}$ dos operadores primários e a 3a.coluna, suas dimensões de escala. A 4 a.coluna mostra os "gaps" em unidades de $\pi / L$ e a última coluna indica as propriedades de simetria $Z(2)$. 


\section{APÊNDICE A \\ REGRAS DE SOMA DE INVERSÃO}

Considere a função de partição para uma teoria definida num retângulo $l \times l^{\prime}$ com condições toroidais de contorno, no limite em que $\ell, l^{\prime} \rightarrow \infty$ com $\ell^{\prime} / \ell=\delta$, fixo.

$$
Z\left(l, l^{\prime}\right)=T_{R} e^{-l^{\prime} \hat{H} p} \text {. }
$$

Os autovalores $\mathrm{E}_{\mathrm{n}}$ de $\hat{\mathrm{H}} \mathrm{p}$ são relacionados às dimensões de escala $x_{n}$ dos operadores de escala da teoria por

$$
E_{n}-E_{0} N \frac{2 \pi x_{n}}{\ell}
$$

e a correção devido ao tamanho finito do sistema ao autovalor mais baixo $E_{0}$ é relacionado à anomalia conforme $\underline{c}$, atravēs de

$$
E_{0} \sim f l-\frac{\pi C}{b L}
$$

onde $f$ é o valor da energia do estado fundamental por sítio no lim 1 te termodinâmico $(1 \rightarrow \infty)$.

Usando (A.2) e (A.3) a relação (A.1) para a função de pạ tição $z\left(l, l^{\prime}\right)$ pode ser escrita na forma

$$
z\left(l, l^{\prime}\right)=e^{\frac{\pi c \delta}{6}} \sum_{n} e^{-\left(E_{n}-E_{0}\right)}
$$

onde consideramos $f=0$. Essa soma sobre $n$ pode ser quebrada em uma soma sobre os blocos conforme e uma outra sobre os operadores de ca da bloco. No nível $(\mathrm{N}, \overline{\mathrm{N}})$ de um bloco, o operador geral tem a forma 


$$
L_{-K_{1}} \ldots L_{-K_{m}} L_{-K_{1}^{\prime}} \ldots \bar{L}_{-K_{m}^{\prime}} \phi
$$

onde $K_{1} \leqslant \ldots \leqslant K_{m}, K_{1} \leqslant \ldots \leqslant K_{m}^{\prime}$ e $\sum K_{j}=N, \sum K_{j}^{\prime}=\bar{N}$, e sua dimensäo de escala é

$$
x=h_{p, q}+\bar{h}_{\bar{p}, \bar{q}}+N+\bar{N}
$$

Existem $P(N) P(\bar{N})$ operadores desse tipo, onde $P(N) \vec{e} \circ n \bar{u}-$ mero de partições de $\mathrm{N}$ em inteiros positivos, não necessariamente distintos. Entretanto, alguns desses operadores podem não ser independentes, o que faz com que a degenerescēncia do nível $(N, \overline{\mathrm{N}})$ näo se ja $P(N) P(\bar{N})$. A soma na equação (4) pode ser escrita na forma

$$
\begin{aligned}
\sum_{N, \bar{N}} \sum_{p q, \bar{p} \bar{q}} d(p, q ; \bar{p}, \bar{q}) d_{p q}(N) d \bar{p} \bar{q}(\bar{N}) \times \\
x \exp \left(-2 \pi \delta\left(h_{p, q}+\bar{h}_{\bar{p}, \bar{q}}+N+\bar{N}\right)\right.
\end{aligned}
$$

onde $d$ é a degenerescência do nível $N$ no bloco conforme e $N(p, q$; $\bar{p}, \bar{q})$ é o número de operadores primārios com $h_{h} h_{p, q}$ e $\bar{h}=h_{\overline{p q}}$. Essa equação tem a mesma forma da förmula de caracteres para a apropriada representação da ālgebra de Virasoro. Estas förmulas foram obtidas por Rocha-Caridı e, são dadas por

$$
Z(\delta)=e^{\frac{\pi c \delta}{b}} \sum_{p q ; \bar{p} \bar{q}} d(p, q ; \bar{p}, \bar{q}) x_{p q}(\delta) x_{\bar{p} \bar{q}}(\delta)
$$

onde 


$$
\begin{aligned}
X_{p q}(\delta)= & \prod_{n=1}^{\infty}\left(1-e^{-2 \pi n \delta}\right)^{-1} \times \sum_{k=-\infty}^{\infty}\left\{\operatorname { e x p } \left(-\frac{\pi \delta}{2 m(m+1)} \times\right.\right. \\
& \left.\left.x\left[(2 m(m+1) k+(m+1) p-m q)^{2}-1\right]\right)-\{q \rightarrow-q\}\right]
\end{aligned}
$$

Com o auxilio da fórmula de soma de poisson, $X_{p, q}$ pode ser colocado na forma

$$
\begin{aligned}
x_{p q}(\delta)= & \left(\frac{2}{m(m+1)}\right)^{1 / 2} \exp \left[\frac{\pi c}{12}\left(\delta^{-1}-\delta\right)\right] \prod_{n=1}^{\infty}\left(1-e^{-2 \pi n \delta^{-1}}\right)^{-1} \\
& \times \sum_{r=-\infty}^{\infty} \exp \left(-\frac{\pi\left(r^{2}-1\right)}{2 \delta m(m+1)}\right) \operatorname{sen} \frac{\pi r p}{m} \operatorname{sen} \frac{\pi r q}{m+1},
\end{aligned}
$$

que com um pouco de manipulação algébrłlca permite reescrever $(A, 6)$ na forma

$$
\begin{aligned}
& Z(\delta)=e^{\frac{\pi c}{\delta \delta}} \sum_{p q, \bar{p} \bar{q}} \sum_{p^{\prime} q^{\prime}, \overline{p^{\prime}} \bar{q}} M_{p q}^{p^{\prime} q^{\prime}} M_{\frac{p^{\prime}}{\bar{p}^{\prime} \bar{q}}}^{\bar{q}} \mathcal{N}\left(p^{\prime} \cdot q^{\prime} ; \bar{p}^{\prime}, \overline{q^{\prime}}\right) \times \\
& x x_{p q}\left(\delta^{-1}\right) x_{p \bar{q}}\left(\delta^{-1}\right)
\end{aligned}
$$

onde os elementos da matriz M são dados por

$$
M_{p q^{\prime}}^{p^{\prime}}=\left(\frac{1}{8} m(m+1)\right)^{1 / 2}(-1)^{(p+q)\left(p^{\prime}+q^{\prime}\right)} \operatorname{sen} \frac{\pi p p^{\prime}}{m} \operatorname{sen} \frac{\pi q q^{\prime}}{m+1}
$$


As regras de soma de inversão são obtidas impondo-se a condição que $z(\delta)=z\left(\delta^{-1}\right)$ e igualando-se potências de $e^{-1 / \delta}, \quad 0$ que resulta em equações lineares para $\mathcal{N}$, escritas como

$$
[M \otimes M] d=S
$$

Como se pode ver de (A.13l, nōs estamos interessados em autovetores de $[M \otimes M]$ com autovalor um. Em geral, este autoespaço é multidimensional e, como queremos apenas soluções nas quais $\mathcal{N}(p, q ; \bar{p}, \bar{q})$ sejam inteiros não negativos, obter as soluções de (A.13) se torna um trabalho difícil. Uma solução que pode sempre ser encontrada é

$$
\mathcal{S}(p, q ; \bar{p}, \bar{q})=\delta p, \bar{p} \delta q, \bar{q}
$$

que corresponde ao caso de todos os operadores escalares possiveis estarem presentes e mais nenhum outro.

Vejamos, por exemplo, o modelo de Ising $(m=3)$ cujos valores permitidos de $h$ e $\bar{h}$ são $0,1 / 16,1 / 2$. Truncada ao espaço de ope radores escalares, a matriz $[M \otimes M]$, com elementos dados por (A.12) é

$$
M=\frac{1}{4}\left(\begin{array}{lll}
1 & 1 & 2 \\
1 & 1 & 2 \\
2 & 2 & 0
\end{array}\right)
$$

Pode-se mostrar daí que a única solução possivel é da for ma (A.14). Os três operadores escalares primários nesse caso são: o operador unidade, a densidade de energia e a magnetização. 


\section{APENDICE B}

\section{O METODO DE LANCZOS}

O algoritmo de Lanczos para diagonalizaçào de matrizes consiste em achar, por construção; uma base na qual o Hamiltoniano tenha uma representação tridiagonal. Isso significa que ao invês de armazenar $q^{N} \times q^{N}$ elementos teremos que guardar apenas $2 q^{N}$, onde $q \quad e$ o nümero de estados por sítio e $\mathrm{N}$ o nümero de sítios.

Para construir a nova base nós escolhemos um estado ini cial $\psi_{0}$ normalizado e a seguir aplicamos $\mathrm{H}$ repetidas vezes. Após a primeira aplicação teremos

$$
H \psi_{0}=a_{1} \psi_{0}+b_{1} \psi_{1}
$$

onde $a_{1}$ e $b_{1}$ são escolhidos de modo que $\psi_{1}$ também seja normallzado e ortogonal a $\psi_{0}$. A seguir atuamos com $\mathrm{H} \mathrm{em} \psi_{1}$ e obtemos

$$
H \psi_{1}=c_{1} \psi_{0}+a_{2} \psi_{1}+b_{2} \psi_{2}
$$

onde $c_{1}, a_{2}$ e $b_{2}$ são escolhidos de maneira que $\Psi_{2}$ seja normalizado e ortogonal a ambos $\psi_{1}$ e $\psi_{2}$. O método prossegue nessa linha, e cada vez que um novo estado é gerado $\left(\psi_{n}\right)$ nós calculamos $H \psi_{n}$, sub traimos as suas componentes ao longo dos outros estados $1 \psi_{0}, \psi_{1}, \ldots$ $\left.\psi_{n}\right)$ e normalizamos o que sobra $\left(\psi_{n+1}\right)$. De forma geral teríamos:

$$
H \psi_{n}=\sum_{i=1}^{n-2} \alpha_{i} \psi_{i}+c_{n} \psi_{n-1}+a_{n+1} \psi_{n}+b_{n+1} \psi_{n+1}
$$

Os $n+1$ estados construídos atē agora são, por construção, ortonormais, e se nós calcularmos a projeção de $\mathrm{H} \psi_{n}$ sobre um estado $\psi_{j}(j\langle n-2)$ encontraremos 


$$
\left(\psi_{j}, H \psi_{n}\right)=\alpha_{j} \ldots
$$

Como $\mathrm{H}$ é hermitiano podemos reescrever (B.4) como

$$
\left(H \psi_{j}, \psi_{n}\right)=\alpha_{j}
$$

Entretanto, usando (B.3) para $n=j$ observamos que $H \Psi_{j}$ não contēm $\psi_{n}$ e portanto

$$
\alpha_{j}=0, \quad \forall j<n-2
$$

Dessa forma concluimos que a partir de $n=1$ teremos

$$
H \psi_{n}=c_{n} \psi_{n-1}+a_{n+1} \psi_{n}+b_{n+1} \psi_{n+1},
$$

e o processo termina quando

$$
H \psi_{\tilde{N}}=c_{\tilde{N}} \psi_{\tilde{N}-1}+a_{\tilde{N}+1} \psi_{\tilde{N}}
$$

ou seja quando $\mathrm{b}_{\tilde{\mathrm{N}}+1}=0$.

A hermiticidade de $\mathrm{H}$ também implica que

$$
c_{n}=b_{n}, \quad n=1,2, \ldots
$$

e, assim, H é escrito na nova base como

$$
H_{T}=\left(\begin{array}{cccccc}
a_{1} & b_{1} & & & 0 & \\
b_{1} & a_{2} & b_{2} & & & \\
& b_{2} & a_{3} & \cdots & & \\
0 & & & & \ddots & a_{\tilde{N}}
\end{array}\right)
$$


A característica principàl desse método è que não hã ne cessidade de completar o processo pafa se obter os autovalores ex tremos de $\mathrm{H}$ com boa precisão. A experiencia mostra que os autovalores mais baixos da submatriz de dimensäo $K<\tilde{N}$, convergem rapidamente para aqueles de H. Essa convergência pode ser monitorada fazendo-se a diagonalização das submatrizes a cada passo e comparando os autovalores de uma iteração com aqueles da anterior. No caso de Hamilto nianos com invariância translacional é possivel acelerar o processo utilizando estados que explorar essa simetria. Em geral consegue se prē-diagonalizar o Hamiltoniano reconhecendo quantidades conservadas como a carga no modelo de potts ou o spin total no modelo Xxz $\left(\Sigma_{i} \sigma^{2}\right)$

Os estados representativos do sistema podem ser guarda dos como números inteiros, O que facilita a aplicação de $\mathrm{H}$ no compu tador. Na linguagem binâría um número inteiro ê uma sequência de uns e zeros que serão associados a spin "up" e spin "down". A aplicação dos operadores é feita através de funções que trabalham diretamente com os bits.

- método de Lanczos pode ser generalizado para hamiltonianos não-hermitianos. Neste caso, procuramos a transformação de similaridade

$$
X^{-1} H X=T
$$

que leva o hamiltoniano $\mathrm{H}$ a uma representação $\mathrm{T}$ tridiagonal e simétrica

$$
T=\left(\begin{array}{cccc}
a_{1} & b_{1} & & 0 \\
b_{1} & a_{2} & b_{2} & \\
& b_{2} & a_{3} & \\
0 & & & \ddots
\end{array}\right)
$$


67

A matriz, X è escrita como uma série de vetores coluna

$\Psi_{1}:$

$$
X=\left(\psi_{0}, \psi_{1}, \ldots, \psi_{N}\right)
$$

As equações do método de Lanczos são as mesmas, sō que agora os coeficientes $c_{n}, a_{n+1} e b_{n+1}$ são números complexos

$$
\begin{aligned}
& H \psi_{0}=a_{1} \psi_{0}+b_{1} \psi_{1} \\
& H \psi_{n}=c_{n} \psi_{n-1}+a_{n+1} \psi_{n}+b_{n+1} \psi_{n+1}
\end{aligned}
$$

Devido à não-hermiticidade de $H, X$ não é unitāria e, assim precisamos gerar sua inversa $x^{-1}$

$$
y=x^{-1}, \quad H^{+} y=y T^{+}, \quad y^{+} x=1
$$

As colunas $\phi_{1}$ de $Y$ podem ser calculadas com as seguintes equações adictona1s

$$
\begin{aligned}
& H^{+} \phi_{0}=a_{1}^{*} \phi_{0}+b_{1}^{*} \phi_{1} \\
& H^{+} \phi_{n}=c_{n}^{*} \phi_{n-1}+a_{n+1}^{*} \phi_{n}+b_{n+1}^{*} \phi_{n+1}
\end{aligned}
$$

$(B, 16)$

de forma que

$$
\phi_{i}^{+} \psi_{j}=\delta_{i j}
$$

Os coeficientes $a_{n+1}$ são, então, obtidos de:

$$
a_{n+1}=\phi_{n}^{+} H \gamma_{n}
$$


e usando que

$$
\begin{aligned}
& b_{n+1} \psi_{n+1}=H \psi_{n}-a_{n+1} \psi_{n}-c_{n} \psi_{n-1} \\
& b_{n+1}^{*} \phi_{n+1}=H^{+} \phi_{n}-a_{n+1}^{*} \phi_{n}-c_{n}^{*} \phi_{n-1}
\end{aligned}
$$

obtém-se os coeficientes $b_{n+1}$ e os vetores de Lanczos $\psi_{n+1}, \phi_{n+1}$

$$
\left(b_{n+1}\right)^{2}=\left(b_{n+1}^{*} \phi_{n-1}\right)^{+}\left(b_{n+1} \psi_{n+1}\right)
$$

Nós implementamos esse esquema no computador com a finalidade de estudar o modelo de Potts-3 quiral. Os resultados estão sendo analisados. 
REFERENCIAS

1) A.M.Polyakov. Sov.Phys.JETP Letters, 12, 381 (1970).

2) J.M.Luck. J.Physc., A15, L169 (1982).

3) B.Derrida e J.de Sèze. J.de Physique 43 , 475 (1982).

4) M.P.Nightingale e H.Blote. J.Phys, Al6, L657 (1985).

5) V.Privman e M.E.Fisher. Phys.Rev. B30, 322 (1984).

6) J.L.Cardy. J.Phys., Al7, L385 (1984).

7) J.L.Cardy. J.Phys., Al7, L961 (1984).

8) A.Belavin, A.Polyakov e A.zamolodchikov. Nucl.Phys. B241, $333(1984)$.

9) V.G.Kac, em "Lectures Notes in Physics", vo1.94, p.441 (Springer-Verlag, New York).

10) D.Frledan, Z.Q1u e S,Shenker. Phys.Rev.Letters, 52, 1575 (1984).

11) J.Cardy, em "Phase Transitions and Critical Phenomena", vol.

11 da Coleção DOMB \& LEBONITz (Academic, New York, 1986).

12) H.Blote, J.Cardy e M.Nightingale. Phys.Rev.Letters $\underline{56}$, $742(1986)$.

I.Affleck. Phys.Rev.Letters 56, 746 (1986).

13) J.B.Kogut. Rev.Mod.Phys., 51, 659 (1979).

14) C.J.Hammer e M.N.Barber. J.PhYs., A13, L169 (1980).

15) Roomany, Wild e Holloway. Phys. Rev.; D21, 1557 (1980).

16) M.Blume. Phys.Rev., 141, 517 (1966).

17) H.Capel. Physica, 32, 966 (1966).

18) Z.Qiu. Nucl.Physics, B270, 205 (1986).

19) E.Lieb, T.Schultz e D.Mattis. Ann.Phys., NY 16, 407(1961). T.W.Burkhardt e I.Guim. J.Phys., A18, L33 (1985). 
20) B.Nienhuis, E.Riedel e M.Schick. J.Phys.,A13, L189(1980).

21) G.Von Gehlen e V.Rittenberg. J.Phys.,Al9, L625 (1986).

$$
\text { J.Phys. , A19, L631 (1986). }
$$

22) J.Cardy. Nucl.Phys., B275, 200 (1986).

23) J.Cardy. Nucl.Phys., B270, 186 (1986).

24) M.Blume, V.Emery e R.Griffths: Phys.Rev. A4, 1071 (1971).

25) H.Hamber. Phys, Rev., B21, 3999 (1980).

26) P.Boyanovshy e L.Masper1. Phys.Rev.,D21, 1550 (1980).

27) C.Lanczos. J.Res. NBS 45,255 (1950).

28) F.Alcaraz, J.R.Drugowlch de Fellcio, R.Kuberle e J.F.

$$
\text { Stilck. Phys,Rev., B32, 7469 (1985). }
$$

29) K.Binder, em "Phase Transitions and Critical Phenomena". , vol. 8 (C.DOMB e J.L.LEBOWITZ, eds.), Academic, London.

30) J.L.Cardy. Nucl.Phys., B240, 514 (1984).

31) B.Nienhuis, E.Riedel e M.Schick. Phys.Rev.Letters, 43 , 737 (1979). 